\title{
Explicit no arbitrage domain for sub-SVIs via reparametrization
}

\author{
Claude Martini*1 and Arianna Mingone ${ }^{\dagger 1,2}$ \\ ${ }^{1}$ Zeliade Systems, 56 rue Jean-Jacques Rousseau, Paris, France \\ ${ }^{2}$ Centre de Mathématiques Appliquées (CMAP), CNRS, Ecole Polytechnique, \\ Institut Polytechnique de Paris
}

June 7, 2021

\begin{abstract}
The no Butterfly arbitrage domain of Gatheral SVI 5-parameters formula for the volatility smile has been recently described. It requires in general a numerical minimization of 2 functions altogether with a few root finding procedures. We study here the case of some sub-SVIs (all with 3 parameters): the Symmetric SVI, the Vanishing Upward/Downward SVI, and SSVI, for which we provide an explicit domain, with no numerical procedure required.
\end{abstract}

\section{Introduction}

Gatheral SVI parametrization of a volatility smile reads:

$$
w(k)=a+b\left(\rho(k-m)+\sqrt{(k-m)^{2}+\sigma^{2}}\right)
$$

where $w(k)$ is the so-called total variance: the squared implied volatility times the remaining time to maturity at the log-forward moneyness $k$.

SVI is known to fit very well a large set of market data, and recently the challenging question of characterizing no Butterfly arbitrage in SVI has been solved with a handy parametrization of the no arbitrage domain, leading to an efficient implementation of a calibration algorithm ensuring no Butterfly arbitrage ([6]). So why would one care about sub-SVIs, meaning SVI with some parameters frozen, or re-parametrizations of SVI with less than 5 parameters, like the volatility slices of SSVI surfaces of Gatheral and Jacquier (3] $)$ ?

In fact, for several reasons. The 1st one is that SVI might be too rich in the sense that an excellent fit could also be achieved in most cases by sub-SVIs, with the additional benefit to stabilize the variation of the calibrated optimal parameter from one day to another, or between different maturity slices. A good theoretical reason to suspect this follows from considering SVI

*cmartini@zeliade.com

†arianna.mingone@polytechnique.edu 
smiles with $\rho=0$ and $m \neq 0$ : indeed the correspondence with stochastic volatility models dictates that models with a zero correlation should yield symmetric smiles, which implies $m=0$; in this sense SVI smiles with $\rho=0$ and $m \neq 0$ should correspond to smiles which are not associated to stochastic volatility models, and also probably not very likely to be met in real-life market data. In this direction, one could also note that the result by Gatheral and Jacquier (2]) that the Long Term Heston smile goes to SVI shows in fact that it goes to a sub-SVI; indeed the SVI parameters are given by

$$
a=\frac{\theta}{2}\left(1-\rho^{2}\right), \quad b=\frac{\theta \varphi}{2}, \quad \quad \quad \quad, \quad=-\frac{\rho}{\varphi}, \quad \sigma=\frac{\sqrt{1-\rho^{2}}}{\varphi},
$$

so that the long-term smile depends eventually only on the 3 parameters $(\theta, \varphi, \rho)$, with the constraint $\rho=0 \Longrightarrow m=0$ enforced.

The 2nd reason is that it is difficult to obtain no Calendar Spread arbitrage conditions on two SVI smiles attached to two different maturities, as discussed in 3. This has been achieved for smiles corresponding to SSVI parameters, which are sub-SVI ones with 3 parameters instead of 5 , as shown in [5. So in order to obtain tractable no arbitrage SVI surfaces, it may be required in practice to restrict the set of SVI parameters. Note that, in relation to the 1st point above, given the poor ability of SSVI to fit especially on the short term, the right balance between fitting ability and tractability might lie in-between SVI and SSVI, in some sub-SVI with 4 parameters.

Another hope is that there might be simplifications, due to the special structure of the different sub-SVIs under study, both in the Fukasawa domain which characterizes a weak no Butterfly arbitrage property and constitutes a key step in the full characterization of no Butterfly arbitrage, and in the last stage of the algorithm in [6], which requires 2 minimizations to get the lower bound of the no arbitrage domain for the SVI parameter $\sigma$. We recall that the computation of the boundary of the Fukasawa domain, which corresponds to the weak necessary condition of no arbitrage obtained by... Fukasawa (1), requires also some root-finding algorithm in general, much quicker than the last stage minimization algorithms for $\sigma$ though.

In this work we study in detail the following $s u b-S V I s$ :

1. The Vanishing $S V I$ where $a=0$ and $\rho= \pm 1$, with its 2 flavors Vanishing upward $(\rho=1)$, and Vanishing downward $(\rho=-1)$; the second family may correspond to real-life smiles and is given by

$$
w(k)=b\left(-(k-m)+\sqrt{(k-m)^{2}+\sigma^{2}}\right)
$$

2. The Symmetric SVI where $\rho=m=0$, so that:

$$
w(k)=a+b \sqrt{k^{2}+\sigma^{2}}
$$

3. SSVI (slices) given by:

$$
w(k)=\frac{\theta}{2}\left(1+\rho \varphi k+\sqrt{(\varphi k+\rho)^{2}+1-\rho^{2}}\right)
$$

For all those sub-SVIs, our program is clear: trying to find out an explicit parametrization for the Fukasawa domain and, when possible, of the full no arbitrage domain. Besides brute-force calculus (that we do use in many circumstances), we heavily use a re-parametrization paradigm, that we illustrate below in the context of the bound on $\sigma$ discussed above. 
This stage requires indeed to compute the minimum of a function $\tilde{f}(l ; \gamma, b, \rho, \mu)$, on an interval which depends on the parameters $(\gamma, \rho)$; we know that $\tilde{f}$ goes to infinity at the bounds of this interval. Our strategy is then to study the critical points of $\tilde{f}$. It turns out that in full generality, the equation characterizing those critical points $\bar{l}$ reads:

$$
p(\bar{l} ; \gamma, \rho, \mu)=b^{2} q(\bar{l} ; \gamma, \rho, \mu)
$$

In some circumstances, we can then use $\bar{l}$ as a parameter, and obtain $b^{2}$ as $\frac{p(\bar{l} ; \gamma, \rho, \mu)}{q(\bar{l} ; \gamma, \rho, \mu)}$. In other circumstances, we use the fact that the equation $p-b^{2} q=0$ is, in full generality, quadratic in $\mu$, and we use the same trick to back up $\mu$ once $\bar{l}$ is promoted to the status of a parameter.

Now this is only the easy part of the story, since the critical points of $\tilde{f}$ may correspond to other local minima than the absolute one, or, even worse, to local maxima. The hard part is to show that the chosen critical point $\bar{l}$, in a given domain, corresponds indeed to the global minimum of $\tilde{f}$; one way to prove this is that there is a unique solution to the critical points equation above. We manage to prove this unicity for the Symmetric and Vanishing case, and resort to a numerical proof for SSVI.

This leads us to the following results:

- in section 3, we obtain a fully explicit parametrization of the no arbitrage domain for the Vanishing Upward and the Vanishing Downward SVI (Propositions 3.2 and 3.4);

- in section 4 we find a parametrization of the no arbitrage domain for the Extremal Decorrelated SVI (Proposition 4.2);

- in section 5, we get the no arbitrage domain for the Symmetric SVI (Proposition 5.2);

- in section 6, we derive a quasi-explicit parametrization (modulo a zero of a one-dimensional function to be computed numerically) of the no arbitrage domain for SSVI (Proposition 6.3). We also re-visit the Long Term Heston SVI appoximation and show it is in fact of SSVI type; we prove in Proposition 6.8 that it is indeed free of Butterfly arbitrage as soon as $T$ is larger than some fully explicit threshold, which completes Gatheral and Jacquier result.

Up to now and to the best of our knowledge, the only known volatility model (meaning: a formula for the implied volatility) with an explicit no arbitrage domain was the SSVI slice, with the (restrictive) conditions obtained by Gatheral and Jacquier ([3]), and extended to the characterization of the full no arbitrage domain in the decorrelated case in [4]. To this extent, the present work is a big leap forward, since we obtain 3 new families: the Vanishing Downward/Upward one, the Symmetric one, and the correlated SSVI, with explicit no arbitrage domains (a single-variable boundary function has to be computed numerically for SSVI).

All our sub-SVIs are 3 parameters SVI; as discussed above, 3 parameters may be too little to produce a good fit on market data, so we would say that the interest here is essentially of academic nature: we hope that those families can help in the investigation of the theoretical properties of volatility smiles, or come as handy illustrations of those properties. Note though that the Vanishing Downward SVI could have a practical application to the case of decreasing market smiles which are often encountered for not-too-short maturities. SSVI is used in practice; our investigation yields a parametrization of the SSVI slices satisfying the no Butterfly arbitrage property which is much more effective than the generic one presented in [6].

We warmly thank Stefano De Marco and Antoine Jacquier for their remarks and comments on a preliminary version of this work. 


\section{Notations and preliminaries}

\subsection{Necessary and sufficient no Butterfly arbitrage conditions for SVI}

In the whole article, we will refer to the results in [6], that we quickly summarize here, with some changes in notations.

The general form for SVI is

$$
S V I(k)=a+b\left(\rho(k-m)+\sqrt{(k-m)^{2}+\sigma^{2}}\right)
$$

where $a, m \in \mathbb{R}, b \geq 0, \rho \in[-1,1], \sigma \geq 0$. If $b=0$ and $a>0$, SVI reduces to the Black-Scholes case, which is free of arbitrage, while if $a=b=0$, we recover the trivial case. In the following we will consider $b>0$.

We set $\gamma=\frac{a}{b \sigma}$ and $\mu=\frac{m}{\sigma}$. Let us redefine the quantity

$$
N(l ; \gamma, \rho):=\gamma+\rho l+\sqrt{l^{2}+1}
$$

such that $S V I(k)=b \sigma N\left(\frac{k-m}{\sigma}\right)$. The derivatives of $N$ are

$$
N^{\prime}(l ; \rho)=\rho+\frac{l}{\sqrt{l^{2}+1}}, \quad \quad N^{\prime \prime}(l)=\frac{1}{\left(l^{2}+1\right)^{\frac{3}{2}}}
$$

so that $N$ has a unique critical point which is a point of minimum equal to $l^{*}=-\frac{\rho}{\sqrt{1-\rho^{2}}}$. Since $N$ is a rescaled total variance, it must be positive, so the constraint on the new variables are $\gamma \geq-\sqrt{1-\rho^{2}}, b \geq 0, \rho \in[-1,1], \mu \in \mathbb{R}, \sigma \geq 0$. When $b=0$, we recover the Black-Scholes case, which is free of Butterfly arbitrage under $\sigma>0$. Then, when formulating theorems of non-arbitrage, we consider only the more difficult cases $b>0$.

We define

$$
\begin{aligned}
h(l ; \gamma, \rho, \mu) & :=1-N^{\prime}(l ; \rho) \frac{l+\mu}{2 N(l ; \gamma, \rho)}, \\
g(l ; \rho) & :=\frac{N^{\prime}(l ; \rho)}{4}, \\
g_{2}(l ; \gamma, \rho) & :=N^{\prime \prime}(l)-\frac{N^{\prime}(l ; \rho)^{2}}{2 N(l ; \gamma, \rho)},
\end{aligned}
$$

and $G_{1}(l):=G_{1+}(l) G_{1-}(l):=(h(l)-b g(l))(h(l)+b g(l))$.

In section 4.1 of [6], it is shown that the requirement that an SVI is (Butterfly) arbitrage-free is equivalent to the requirement that the function

$$
G_{1}+\frac{1}{2 \sigma} b g_{2}
$$

is non-negative. Fukasawa proved in [1] that the condition $G_{1+}>0$ and $G_{1-}>0$ are also necessary. Theorem 5.10 of [6], that we rewrite here, characterizes these conditions in the case of SVI. The statement requires the definition of the functions:

$$
\begin{aligned}
L_{-}(l ; \gamma, b, \rho) & :=2 N(l ; \gamma, \rho)\left(\frac{1}{N^{\prime}(l ; \rho)}+\frac{b}{4}\right)-l, \\
g_{-(b, \rho)} & :=\left(\rho \sqrt{l^{2}+1}+l\right)^{2}\left(\sqrt{l^{2}+1}\left(\frac{1}{2}+\frac{b \rho}{4}\right)+\frac{b l}{4}\right)-\left(\rho l+\sqrt{l^{2}+1}\right) .
\end{aligned}
$$


When $\gamma+\sqrt{1-\rho^{2}}>0$ and $|\rho|<1$, preliminary propositions show that under the case $b(1 \pm \rho)<2$, there exist a unique $l_{-}(\gamma, b, \rho)<l^{*}$ and a unique $l_{-}(\gamma, b,-\rho)<l^{*}$ such that $g_{-(b, \rho)}\left(l_{-}(\gamma, b, \rho)\right)=\gamma$ and $g_{-(b,-\rho)}\left(l_{-}(\gamma, b,-\rho)\right)=\gamma$. In such way, the quantity

$$
\tilde{F}(b, \rho):=\inf \left\{\gamma \mid-L_{-}\left(l_{-}(\gamma, b,-\rho) ; \gamma, b,-\rho\right)>L_{-}\left(l_{-}(\gamma, b, \rho) ; \gamma, b, \rho\right)\right\} \wedge-\sqrt{1-\rho^{2}}
$$

is well-defined and it is called the Fukasawa threshold. If instead $b(1-\rho)=2($ or $b(1+\rho)=$ $2)$, there exists a unique $l_{-}(\gamma, b,-\rho)<l^{*}\left(\right.$ resp. $\left.l_{-}(\gamma, b, \rho)\right)$ such that $g_{-(b,-\rho)}\left(l_{-}\right)=\gamma$ (resp. $\left.g_{-(b, \rho)}\left(l_{-}\right)=\gamma\right)$. When the former case arises while the latter does not, the Fukasawa threshold is defined as $\tilde{F}(b, \rho):=\inf \left\{\gamma \mid-L_{-}\left(l_{-}(\gamma, b,-\rho) ; \gamma, b,-\rho\right)>-\frac{b \gamma}{2}\right\} \wedge-\sqrt{1-\rho^{2}}$. Vice versa, when it is the second case to be active while the first is not, the quantity is defined as $\tilde{F}(b, \rho):=\inf \left\{\gamma \mid \frac{b \gamma}{2}>\right.$ $\left.L_{-}\left(l_{-}(\gamma, b, \rho) ; \gamma, b, \rho\right)\right\} \wedge-\sqrt{1-\rho^{2}}$. Finally, when both cases are valid, so $b=2$ and $\rho=0$, the threshold is $\tilde{F}(2,0):=0$. The aforementioned theorem is:

Theorem 2.1 (SVI parameters $(\gamma, b, \rho, \mu, \sigma)$ fulfilling Fukasawa necessary no arbitrage conditions). Assume $\gamma+\sqrt{1-\rho^{2}}>0$ and $|\rho|<1$. Then:

- if $b(1 \pm \rho)<2$, it holds that $\tilde{F}(b, \rho)<0$ and the interval $\left.I_{\gamma, b, \rho}=\right] L_{-}\left(l_{-}(\gamma, b, \rho) ; \gamma, b, \rho\right)$, $-L_{-}\left(l_{-}(\gamma, b,-\rho) ; \gamma, b,-\rho\right)[$ is non-empty iff $\gamma>\tilde{F}(b, \rho)$;

- if $b(1-\rho)=2($ or $b(1+\rho)=2)$ and $\rho \neq 0$, it holds that $\tilde{F}(b, \rho)<0$ and the interval $\left.I_{\gamma, b, \rho}=\right]-\frac{b \gamma}{2},-L_{-}\left(l_{-}(\gamma, b,-\rho) ; \gamma, b,-\rho\right)\left[\left(\operatorname{resp} . I_{\gamma, b, \rho}=\right] L_{-}\left(l_{-}(\gamma, b, \rho) ; \gamma, b, \rho\right), \frac{b \gamma}{2}[)\right.$ is nonempty iff $\gamma>\tilde{F}(b, \rho)$;

- if $b=2$ and $\rho=0$, the interval $\left.I_{\gamma, 2,0}=\right]-\gamma, \gamma[$ is non-empty iff $\gamma>\tilde{F}(2,0)=0$.

In every case, the Fukasawa conditions are satisfied iff $\mu \in I_{\gamma, b, \rho}$.

The final necessary and sufficient conditions for no Butterfly arbitrage in the case $\gamma+\sqrt{1-\rho^{2}}>$ 0 and $|\rho|<1$ are summed up in Theorem 6.2 of [6], in which $\sigma^{*}$ is defined as

$$
\sigma^{*}(\gamma, b, \rho, \mu):=\sup _{l<l_{1} \vee l>l_{2}}-\frac{b g_{2}(l)}{2 G_{1}(l)} .
$$

Theorem 2.2 (Necessary and sufficient no Butterfly arbitrage conditions for SVI, $\gamma+\sqrt{1-\rho^{2}}>0$ and $|\rho|<1)$. No Butterfly arbitrage in SVI entails that $G_{1}$ is positive, which requires $b(1 \pm \rho) \leq 2$. Under this condition:

- each of the factors of the function $G_{1}$ is positive on $\mathbb{R}$ if and only if $\gamma>\tilde{F}(b, \rho)$ and $\mu \in I_{\gamma, b, \rho}$;

- for such $\mu$ 's, calling $l_{1}<0<l_{2}$ the only zeros of $g_{2}$, the function $G_{1}+\frac{1}{2 \sigma} b g_{2}$ is positive in ]$l_{1}, l_{2}\left[\right.$ for every $\sigma \geq 0$ and it is non-negative on $\mathbb{R}$ if and only if $\sigma \geq \sigma^{*}(\gamma, b, \rho, \mu)$.

In the case of $|\rho|=1$ and $\gamma \geq 0$, Theorem 6.3 of [6] holds:

Theorem 2.3 (Necessary and sufficient no Butterfly arbitrage conditions for SVI, $\rho=-1$ ). No Butterfly arbitrage in SVI entails that $G_{1}$ is positive, which requires $b \leq 1$ and $\gamma \geq 0$. Under these conditions:

- each of the factors of the function $G_{1}$ is positive on $\mathbb{R}$ if and only if $\mu>L_{-}\left(l_{-} ; \gamma, b,-1\right)$; 
- for such $\mu$ 's, calling $l_{1}<0$ the only zero of $g_{2}$, the function $G_{1}+\frac{1}{2 \sigma} b g_{2}$ is positive on ]$l_{1}, \infty\left[\right.$ for every $\sigma \geq 0$ and it is non-negative on $\mathbb{R}$ if and only if $\sigma \geq \sigma^{*}(\gamma, b,-1, \mu)$ where $\sigma^{*}(\gamma, b,-1, \mu):=\sup _{l<l_{1}}-\frac{b g_{2}(l)}{2 G_{1}(l)}$.

From now on, we denote $f:=-\frac{b g_{2}}{2 G_{1}}$ and $\tilde{f}:=-\frac{G_{1}}{g_{2}(l)}$. In this way, the value of the supremum of $f$ is equal to $b$ times half the reciprocal of the infimum of $\tilde{f}$, and the point at which the supremum of $f$ is reached is exactly the point at which the infimum of $\tilde{f}$ is reached.

We present here some general observations that will be used in the next sections.

In order to calculate $\sigma^{*}$, one should find the supremum of the function $f$ over a domain constituted of two open intervals: on the left of $l_{1}$, the first zero of $g_{2}$, and on the right of $l_{2}$, the second zero of $g_{2}$. From a computational point of view, one needs to perform two maximum searches and to compare the two found values to choose the highest. Sometimes this double search is not necessary, indeed for the sub-SVIs studied in this article, the signs of $\rho$ and $\mu$ determine the interval where the global supremum of $f$ lies. Since $l_{1}<0<l_{2}$, the trick will be to compare the two quantities $f(l)$ and $f(-l)$, but first of all, it is necessary to study the two intervals of interest. In particular, the following Lemma holds:

Lemma 2.4. If $\rho \geq 0$ and $l>0$, then $g_{2}(l) \leq g_{2}(-l)$ and $l_{2}(\gamma, \rho) \leq-l_{1}(\gamma, \rho)$. If $\rho<0$ and $l>0$, then $g_{2}(l)>g_{2}(-l)$ and $l_{1}(\gamma, \rho)>-l_{2}(\gamma, \rho)$.

Proof. Fix $l>0$, then for $\rho \geq 0$, it holds

$$
\begin{aligned}
g_{2}(-l)-g_{2}(l) & =\frac{l \rho\left(\left(l^{2}+1\right)\left(1-\rho^{2}\right)+2 \gamma \sqrt{l^{2}+1}+1\right)}{\left(l^{2}+1\right) N(l) N(-l)} \\
& \geq \frac{l \rho\left(\sqrt{\left(l^{2}+1\right)\left(1-\rho^{2}\right)}-1\right)^{2}}{\left(l^{2}+1\right) N(l) N(-l)} \geq 0
\end{aligned}
$$

since $\gamma \geq-\sqrt{1-\rho^{2}}$. So $g_{2}(l) \leq g_{2}(-l)$ and in particular $g_{2}\left(-l_{1}\right) \leq g_{2}\left(l_{1}\right)=0$ so $l_{2}(\gamma, \rho) \leq$ $-l_{1}(\gamma, \rho)$. These inequalities are strict for $\rho$ strictly positive. Similarly for $\rho<0$, we find $g_{2}(l)>$ $g_{2}(-l)$ and $l_{1}(\gamma, \rho)>-l_{2}(\gamma, \rho)$.

This Lemma has a direct consequence, which is that for $\rho \geq 0$, the supremum of $f$ on the left of $l_{2}$ is higher than its supremum on the left of $-l_{1}$, since in the first case, $f$ could attain it between $l_{2}$ and $-l_{1}: \sup _{l>l_{2}} f(l) \geq \sup _{l>-l_{1}} f(l)$. If we can show that for positive $l \mathrm{~s}, f(l) \geq f(-l)$, then the last term is greater than $\sup _{l>-l_{1}} f(-l)$. Making a change of variable, this quantity is equal to $\sup _{l<l_{1}} f(l)$ so this means that $\sigma^{*}$ can be found as the supremum of $f$ on the right of $l_{2}$.

The request $f(l) \geq f(-l)$ is satisfied if, for example, $G_{1}(l) \leq G_{1}(-l)$ because in the above Lemma we showed $g_{2}(l) \leq g_{2}(-l)$. The difference between $G_{1}(l)$ and $G_{1}(-l)$ can be written as $(h(l)-h(-l))(h(l)+h(-l))-b^{2}(g(l)-g(-l))(g(l)+g(-l))$. The quantity $g(l)-g(-l)$ is equal to $\frac{l}{2 \sqrt{l^{2}+1}}$, which is positive, while $g(l)+g(-l)$ is $\frac{\rho}{2}$, which again is non-negative for $\rho \geq 0$. Showing that the product with the $h$ functions is non-positive would then be enough to reach the desired inequality.

Lemma 2.5. Let $\rho=0$. If $\mu \geq 0$, then $\sigma^{*}(\gamma, b, 0, \mu)=\sup _{l>l_{2}} f(l)$ while if $\mu<0$, then $\sigma^{*}(\gamma, b, 0, \mu)=\sup _{l<l_{1}} f(l)$. 
Proof. In the case $\rho=0$, it holds $l_{2}=-l_{1}$ since $g_{2}$ is symmetric. It can be shown that in general

$$
\begin{aligned}
& h(l)-h(-l)=-\frac{l}{\sqrt{l^{2}+1}} \frac{\rho\left(\gamma \sqrt{l^{2}+1}+1\right)+\mu\left(\gamma+\left(1-\rho^{2}\right) \sqrt{l^{2}+1}\right)}{N(l) N(-l)}, \\
& h(l)+h(-l)=\frac{\left(\gamma \sqrt{l^{2}+1}+1\right)\left(2 \gamma+2 \sqrt{l^{2}+1}-\rho \mu\right)+l^{2}\left(\gamma+\left(1-\rho^{2}\right) \sqrt{l^{2}+1}\right)}{\sqrt{l^{2}+1} N(l) N(-l)} .
\end{aligned}
$$

In particular for $\rho=0$ and positive $l$, the sign of the former quantity is the sign of $-\mu$ while the numerator of the latter quantity is $\left(\gamma+\sqrt{l^{2}+1}\right)\left(2 \gamma \sqrt{l^{2}+1}+l^{2}+2\right)$, which is always positive for $\gamma>-1$. Then $G_{1}(l)-G_{1}(-l)=(h(l)-h(-l))(h(l)+h(-l))$ follows the sign of $-\mu$ and so does $f(-l)-f(l)$. If $\mu$ is non-negative, $f(l) \geq f(-l)$ so its supremum must be searched on the right of $l_{2}$. If $\mu$ is negative, the opposite inequality holds for $f$ and its supremum lies on the left of $l_{1}$.

These Lemmas will be useful for the sub-SVIs studied in this article, however it must be noticed that the inequality $f(l) \geq f(-l)$ does not hold in general.

\subsection{Smile inversion}

Tehranchi proved in [7] that a curve of Call prices (with unit underlyer) parametrized by the strike $\kappa$ is free of Butterfly arbitrage if and only if it is convex and satisfies $1 \geq C(\kappa) \geq(1-\kappa)^{+}$for every $\kappa \geq 0$. Moreover, $C$ has these properties if and only if $C^{*}(\kappa):=1-\kappa+\kappa C\left(\frac{1}{\kappa}\right)$ has them.

Note that if at some point $\kappa$ it holds $C(\kappa)=1$, then since $C$ should be convex and decreasing, then $C$ is the constant function 1. Because this case is uninteresting, we can assume that $1>C(\kappa)$ for every $\kappa$. Then there is a unique total variance function $w(k)$ such that $C_{B S}(\kappa, \sqrt{w(k)})=C(\kappa)$ where $C_{B S}$ is the (normalized) Black-Scholes Call price function, and a unique $w^{*}(k)$ such that $C_{B S}\left(\kappa, \sqrt{w^{*}(k)}\right)=C^{*}(\kappa)$. By the Put-Call parity for the Black-Scholes model it holds that

$$
P_{B S}\left(\kappa, \sqrt{w^{*}(k)}\right)=C_{B S}\left(\kappa, \sqrt{w^{*}(k)}\right)+\kappa-1=\kappa C\left(\frac{1}{\kappa}\right) .
$$

Now the LHS is equal to $\kappa N\left(\frac{k}{\sqrt{w^{*}(k)}}+\frac{\sqrt{w^{*}(k)}}{2}\right)-N\left(\frac{k}{\sqrt{w^{*}(k)}}-\frac{\sqrt{w^{*}(k)}}{2}\right)$ and the RHS is equal to $\kappa\left(N\left(\frac{k}{\sqrt{w(-k)}}+\frac{\sqrt{w(-k)}}{2}\right)-\frac{1}{\kappa} N\left(\frac{k}{\sqrt{w(-k)}}-\frac{\sqrt{w(-k)}}{2}\right)\right)$. By the monotonicity of the function $u \rightarrow C_{B S}(\kappa, u)$ it follows then that $w^{*}(k)=w(-k)$. Eventually, we can reword the symmetry of Tehranchi involution $C \rightarrow C^{*}$ at the implied volatility level: a smile $w$ is free of Butterfly arbitrage if and only if the inverse smile $k \rightarrow w(-k)$ is.

Let us now relate the implied volatility of $C$ with that of $C^{*}$.

In the case of SVI, this reduces to have

$$
a+b\left(\rho(k-m)+\sqrt{(k-m)^{2}+\sigma^{2}}\right)=a^{*}+b^{*}\left(\rho^{*}\left(-k-m^{*}\right)+\sqrt{\left(-k-m^{*}\right)^{2}+\sigma^{* 2}}\right)
$$

for all $k$. We prove that this happens iff $a^{*}=a, b^{*}=b, \rho^{*}=-\rho, m^{*}=-m$ and $\sigma^{*}=\sigma$. Of course, $a^{*}, b^{*}, \rho^{*}, m^{*}$ and $\sigma^{*}$ do not depend on $k^{*}$ so neither on $k$. Making the derivative with respect to $k$ at the latter equality gives

$$
b\left(\rho+\frac{k-m}{\sqrt{(k-m)^{2}+\sigma^{2}}}\right)=b^{*}\left(-\rho^{*}+\frac{k+m^{*}}{\sqrt{\left(k+m^{*}\right)^{2}+\sigma^{* 2}}}\right) .
$$


Evaluating this for $k$ going to $\pm \infty$, it must hold $b(\rho \pm 1)=b^{*}\left(-\rho^{*} \pm 1\right)$ or equivalently $b^{*}=b$ and $\rho^{*}=-\rho$. In the same equation, consider $k=m$, then it must hold

$$
b \rho=b\left(\rho+\frac{m+m^{*}}{\sqrt{\left(m+m^{*}\right)^{2}+\sigma^{* 2}}}\right)
$$

which reduces to $m^{*}=-m$. At this point, 3 becomes $\frac{k-m}{\sqrt{(k-m)^{2}+\sigma^{2}}}=\frac{k+m^{*}}{\sqrt{\left(k+m^{*}\right)^{2}+\sigma^{* 2}}}$ so $\sigma^{*}=\sigma$. Finally, from (2), it follows $a^{*}=a$.

Proposition 2.6 (Absence of Butterfly arbitrage for the inverse SVI). The $S V I(a, b, \rho, m, \sigma)$ is Butterfly arbitrage-free iff the $S V I(a, b,-\rho,-m, \sigma)$ is Butterfly arbitrage-free.

\subsection{The $b^{*}$ approach: reparametrizing from the critical point equation}

We now explain how to properly perform the trick described in the Introduction 1 which allows to switch the roles of the parameter $b$ and of the critical point $\bar{l}$ depending on $b$ of $\tilde{f}$. The key passage will be the exploitation of the characteristic equation of these critical points: $h(\bar{l}) p(\bar{l})-b^{2} g(\bar{l}) q(\bar{l})=0$. Three hypothesis must be verified:

1. the Fukasawa bound for $\gamma$ must be a monotone function in $b$;

2. there is uniqueness for the critical points of $\tilde{f}$;

3. the functions $p$ and $q$ do not vanish at the same point.

In $[6$, the Fukasawa conditions read $\gamma>\tilde{F}(b, \rho)$. Firstly, suppose that for fixed $\rho$, the function $b \rightarrow \tilde{F}(b, \rho)$ is monotone and surjective from $\left[0, \frac{2}{1+|\rho|}\right]$ to $[-1,0]$. Then, its inverse $\tilde{F}^{-1}(\cdot, \rho)$ is well defined on $[-1,0]$ and we shall extend it to

$$
\tilde{G}(\gamma, \rho):= \begin{cases}\tilde{F}^{-1}(\gamma, \rho) & \text { if } \gamma \in]-1,0] \\ \frac{2}{1+|\rho|} & \text { if } \gamma>0 .\end{cases}
$$

Secondly, suppose we have proven that for $l>l_{2}$, the function $\tilde{f}$ has exactly one critical point $\bar{l}(\gamma, b, \rho, \mu)$ for $\rho \in[-1,1], \gamma>-1, b \in[0, \tilde{G}(\gamma, \rho)], \mu \in] L_{-}, L_{+}$[. This critical point must then be a local and global point of minimum. Note that we require the uniqueness also for $b=0$ and $b=\tilde{G}(\gamma, \rho)$. This point satisfies $\tilde{f}^{\prime}(\bar{l})=0$ or equivalently

$$
h(\bar{l}) p(\bar{l})-b^{2} g(\bar{l}) q(\bar{l})=0
$$

with $h(\bar{l})=G_{1+}(l)+G_{1-}(l)>0, g(l)>0$ and $p(l):=h(l) g_{2}^{\prime}(l)-2 h^{\prime}(l) g_{2}(l)$ and $q(l):=g(l) g_{2}^{\prime}(l)-$ $2 g^{\prime}(l) g_{2}(l)$.

In particular, either $q(\bar{l})=p(\bar{l})=0$ or

$$
b^{2}=\frac{h(\bar{l})\left(h(\bar{l}) g_{2}^{\prime}(\bar{l})-2 h^{\prime}(\bar{l}) g_{2}(\bar{l})\right)}{g(\bar{l})\left(g(\bar{l}) g_{2}^{\prime}(\bar{l})-2 g^{\prime}(\bar{l}) g_{2}(\bar{l})\right)}
$$

when $q(\bar{l}) \neq 0$. 
It is natural to define on the set $B(\gamma, \rho, \mu)=\{l \in] l_{2}(\gamma, \rho), \infty[: p(l) q(l)>0\}$ a positive function $b^{*}$ by the formula

$$
b^{* 2}(l):=\frac{h(l) p(l)}{g(l) q(l)} .
$$

Note that the function $b^{*}$ might go on $B(\gamma, \rho, \mu)$ to levels not allowed for $b$; yet this function is continuous on its domain of definition and when $q(\bar{l}) \neq 0$ it holds that $b^{2}=b^{* 2}(\bar{l})$. As an immediate consequence, note that if $b_{1} \neq b_{2}$ are such that $\bar{l}\left(\gamma, b_{1}, \rho, \mu\right), \bar{l}\left(\gamma, b_{2}, \rho, \mu\right) \in B(\gamma, \rho, \mu)$, then $\bar{l}\left(\gamma, b_{1}, \rho, \mu\right) \neq \bar{l}\left(\gamma, b_{2}, \rho, \mu\right)$. Note also that we don't know if $B$ is one-piece, i.e. connected.

The question of interest is now the location of the set $Z(\gamma, \rho, \mu):=\{\bar{l}(\gamma, b, \rho, \mu), b \in] 0, \tilde{G}(\gamma, \rho)[\}$ (note that we exclude 0 , this will turn to be more convenient below) with respect to the sets $B(\gamma, \rho, \mu)$ and $\{p=q=0\}$. The third and last hypothesis is that, for the fixed parameters $(\gamma, \rho, \mu)$, the set $\{p=q=0\}$ is empty. Then $Z(\gamma, \rho, \mu)$ is contained in $B(\gamma, \rho, \mu)$. From the above remark, the function $\bar{l}(\gamma, \cdot, \rho, \mu)$ is injective; however at this stage we don't know whether it is continuous (which would imply it is either continuous increasing or continuous decreasing).

Remember that the proof of the uniqueness of the critical point of $\tilde{f}$ still holds for $b=\tilde{G}(\gamma, \rho)$. Then for $\left.(l, b) \in] l_{2}, \infty\right] \times[0, \tilde{G}(\gamma, \rho)]$, the equation

$$
h(l) p(l)-b^{2} g(l) q(l)=0
$$

characterizes the points $\bar{l}(\gamma, b, \rho, \mu)$, since $\tilde{f}$ has a single local and global minimum. As discussed above, in the open set $B(\gamma, \rho, \mu)$, this equation defines a continuous function $b^{*}$; from the characterizing property we get that $Z(\gamma, \rho, \mu)$ eventually coincides with $b^{*-1}(] 0, \tilde{G}(\gamma, \rho)[) \subseteq B(\gamma, \rho, \mu)$, so that in particular $Z(\gamma, \rho, \mu)$ is an open set. Furthermore, the function $b \rightarrow \bar{l}(\gamma, b, \rho, \mu)$ is the inverse of $\left.b^{*}: Z(\gamma, \rho, \mu) \rightarrow\right] 0, \tilde{G}(\gamma, \rho)[$.

It remains to prove that $Z(\gamma, \rho, \mu)$ is one-piece. Indeed, take a sequence $\left(b_{n}\right)_{n}$ such that $\bar{l}_{n}=\bar{l}\left(\gamma, b_{n}, \rho, \mu\right)$ goes to $\bar{l} \in \bar{Z}(\gamma, \rho, \mu)$. Since $\left(b_{n}\right)_{n}$ is a bounded sequence, it has a subsequence converging to a certain $b \in[0, \tilde{G}(\gamma, \rho)]$; call such subsequence as the original one, then the correlated subsequence of $\bar{l}_{n}$ still converges to $\bar{l}$. Since for every $n$ we have $h\left(\bar{l}_{n}\right) p\left(\bar{l}_{n}\right)-b_{n}^{2} g\left(\bar{l}_{n}\right) q\left(\bar{l}_{n}\right)=0$ where all the functions are continuous, then taking the limit, it holds $h(\bar{l}) p(\bar{l})-b^{2} g(\bar{l}) q(\bar{l})=0$. From the characteristic equation above, this yields in turn that $\bar{l}=\bar{l}(\gamma, b, \rho, \mu)$. Also, $b$ is unique because either $b=b^{*}(\bar{l})$ or $p(\bar{l})=q(\bar{l})=0$, but the set $\{p=q=0\}$ is empty. Then it must hold either that $\bar{l} \in Z(\gamma, \rho, \mu)$ or that $\bar{l}$ is the unique critical point $\bar{l}(\gamma, b, \rho, \mu)$ of $\tilde{f}$ when $b=0$ or $b=\tilde{G}(\gamma, \rho)$. The boundary of $Z(\gamma, \rho, \mu)$ is the set $\{\bar{l}(\gamma, 0, \rho, \mu), \bar{l}(\gamma, \tilde{G}(\gamma, \rho), \rho, \mu)\}$ and because $Z(\gamma, \rho, \mu)$ is an open set, we get that $Z(\gamma, \rho, \mu)=] \bar{l}(\gamma, 0, \rho, \mu), \bar{l}(\gamma, \tilde{G}(\gamma, \rho), \rho, \mu)[$ or $Z(\gamma, \rho, \mu)=] \bar{l}(\gamma, \tilde{G}(\gamma, \rho), \rho, \mu), \bar{l}(\gamma, 0, \rho, \mu)[$.

As a consequence we also get that $\bar{l}(\gamma, \cdot, \rho, \mu)$ is either strictly increasing or strictly decreasing, and that $Z(\gamma, \rho, \mu)$ is a single connected component of $B(\gamma, \rho, \mu)$, with a boundary point $\bar{l}(\gamma, 0, \rho, \mu)$ which is the single zero of $p$, and the other boundary point lying within $B(\gamma, \rho, \mu)$.

\section{Vanishing SVI}

\subsection{Vanishing (Upward) SVI}

In this section, we work with the Vanishing Upward SVI and immediately recover the final results on the Vanishing Downward SVI in Section 3.4 


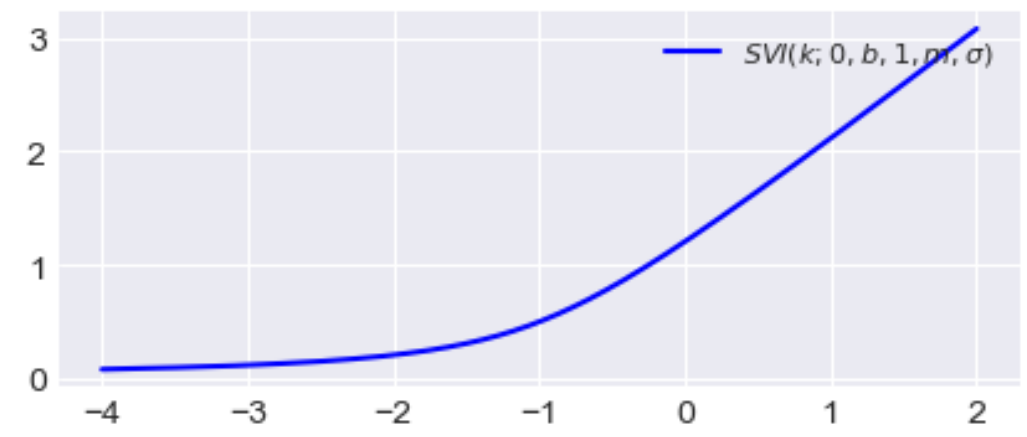

Figure 1: Vanishing Upward SVI with $b=\frac{1}{2}, m=-1$ and $\sigma=1$.

The Vanishing Upward SVI is the sub-SVI obtained by setting $\rho=1$ and $a=0$. The corresponding SVI formula becomes

$$
S V I(k ; 0, b, 1, m, \sigma)=b\left(k-m+\sqrt{(k-m)^{2}+\sigma^{2}}\right) .
$$

With our notations, $N(l)=l+\sqrt{l^{2}+1}$. Note that the Roger Lee conditions require $0<b \leq 1$.

We plot in Figure 1 a Vanishing Upward SVI with $b=\frac{1}{2}, m=-1$ and $\sigma=1$.

The wording Vanishing refers to the fact that the smile goes to 0 on the left, upward meaning it is increasing. The symmetric smile with $\rho=-1$ will be a Vanishing Downward one.

\subsection{The Fukasawa conditions}

Since $\gamma=0$, the Fukasawa condition on $\gamma$ is automatically satisfied. We cite here a result obtained in paragraph 5.3 .1 of $[6]$.

Lemma 3.1 (Fukasawa conditions for the Vanishing Upward SVI). A Vanishing Upward SVI with $0<b \leq 1$ satisfies the Fukasawa conditions iff $\mu<\sqrt{3(1-b)}$.

\subsection{The condition on $\sigma$}

Let us define the two following auxiliary functions:

$$
\begin{aligned}
\mu^{*}(x):= & {\left[2(1-x)\left(2 x^{2}-8 x-1\right)+\right.} \\
& \left.+\sqrt{4 b^{2} x^{6}+8 b^{2} x^{5}+8 x^{4}\left(8-b^{2}\right)-4 x^{3}\left(5 b^{2}+32\right)+x^{2}\left(96-b^{2}\right)+2 x\left(5 b^{2}-16\right)+4+3 b^{2}}\right] \\
& /\left[2 \sqrt{1-x^{2}}\left(2 x^{2}-2 x-1\right)\right], \\
\sigma^{*}(x):= & -\frac{4 b \sqrt{1-x^{2}}\left(1-x-2 x^{2}\right)}{4\left(2-x-\mu^{*}(x) \sqrt{1-x^{2}}\right)^{2}-b^{2}(1+x)^{2}}
\end{aligned}
$$

We show then the following: 
Proposition 3.2 (Fully explicit no arbitrage domain for the Vanishing Upward SVI). A Vanishing Upward SVI with $b=1$ is arbitrage-free iff $\mu<0$ and $\sigma \geq-\frac{\mu}{2}$.

A Vanishing Upward SVI with $0<b<1$ is arbitrage-free iff it can be parametrized as

$$
S V I(k)=b \sigma\left(\frac{k}{\sigma}-\mu^{*}(x)+\sqrt{\left(\frac{k}{\sigma}-\mu^{*}(x)\right)^{2}+1}\right)
$$

where $\frac{2+b}{4-b}<x<1$ and $\sigma \geq \sigma^{*}(x)$.

\subsubsection{Proof of Proposition 3.2}

Note that

$$
\begin{aligned}
G_{1}(l) & =\left(1-\frac{(l+\mu)}{2 \sqrt{l^{2}+1}}\right)^{2}-\frac{b^{2}}{16}\left(1+\frac{l}{\sqrt{l^{2}+1}}\right)^{2}, \\
g_{2}(l) & =\frac{1}{\left(l^{2}+1\right)^{\frac{3}{2}}}-\frac{\sqrt{l^{2}+1}+l}{2\left(l^{2}+1\right)} .
\end{aligned}
$$

The $g_{2}$ function has only one positive zero. Indeed, its zeros solve $2=l^{2}+1+l \sqrt{l^{2}+1}$, or yet $1-l^{2}=l \sqrt{l^{2}+1}$. The only possible solution satisfies $l^{2} \leq 1$, so $l_{2}=\frac{1}{\sqrt{3}}$. In order to have no arbitrage, we then need $\sigma \geq \sigma^{*}=\sup _{l \in] l_{2},+\infty[}-\frac{b g_{2}(l)}{2 G_{1}(l)}$.

From now on, we operate a change of variable setting $x=\frac{l}{\sqrt{l^{2}+1}}$ so that the points $l=l_{2}$ and $l=\infty$ correspond to $x=\frac{1}{2}$ and $x=1$ respectively. Also, $\frac{1}{\sqrt{l^{2}+1}}=\sqrt{1-x^{2}}$.

We call $J_{1}$ and $j_{2}$ the functions $G_{1}$ and $g_{2}$ evaluated at $x$, so

$$
\begin{aligned}
& J_{1}(x)=\left(1-\frac{x}{2}-\frac{\mu}{2} \sqrt{1-x^{2}}\right)^{2}-\frac{b^{2}}{16}(1+x)^{2}, \\
& j_{2}(x)=\frac{\sqrt{1-x^{2}}}{2}\left(1-x-2 x^{2}\right) .
\end{aligned}
$$

The first derivative of $j_{2}$ is $j_{2}^{\prime}(x)=\frac{1}{2 \sqrt{1-x^{2}}}\left(6 x^{3}+2 x^{2}-5 x-1\right)$ which is positive iff $x>\frac{2+\sqrt{10}}{6}:=$ $x_{m_{2}}$. Also, $j_{2}^{\prime \prime}(x)=\frac{-12 x^{4}-2 x^{3}+18 x^{2}+3 x-5}{2\left(1-x^{2}\right)^{\frac{3}{2}}}$ and the only inflection point of $j_{2}$ in $\left[\frac{1}{2}, 1\right]$ is $\frac{1}{2}$. Then, the function $j_{2}$ is null at $\frac{1}{2}$, decreases to its minimum $j_{2}\left(x_{m_{2}}\right)=-\frac{34 \sqrt{2}-5 \sqrt{5}}{108}$ and then increases to 0 for $x=1$, furthermore it is always convex.

Let us turn to the study of $J_{1}$. We have $J_{1}(1)=\frac{1-b^{2}}{4}$ and we know that for the Fukasawa conditions, both $J_{1+}$ and $J_{1-}$ are positive, where $J_{1}=J_{1+} J_{1-}$ and $J_{1 \pm}=1-\frac{x}{2}-\frac{\mu}{2} \sqrt{1-x^{2}} \mp \frac{b}{4}(1+x)$. Considering the first derivatives, we know $J_{1}^{\prime}=J_{1+}^{\prime} J_{1-}+J_{1+} J_{1-}^{\prime}$ where

$$
J_{1 \pm}^{\prime}(x)=-\frac{1}{2}+\frac{\mu}{2} \frac{x}{\sqrt{1-x^{2}}} \mp \frac{b}{4} .
$$

If $\mu \leq 0$, then $J_{1 \pm}$ are decreasing and so is $J_{1}$. In particular, it attains its minimum at $x=1$ and we get that $J=J_{1}+\frac{1}{2 \sigma} b j_{2}$ is always positive for

$$
\sigma \geq-\frac{b j_{2}\left(x_{m_{2}}\right)}{2 J_{1}(1)}=\frac{(34 \sqrt{2}-5 \sqrt{5}) b}{54\left(1-b^{2}\right)}
$$


when $b<1$. We have therefore the explicit no arbitrage sub-domain:

Lemma 3.3 (No arbitrage sub-domain for the Vanishing Upward SVI). A Vanishing Upward SVI with $0<b<1, \mu \leq 0$ and $\sigma \geq \frac{(34 \sqrt{2}-5 \sqrt{5}) b}{54\left(1-b^{2}\right)}$ is arbitrage-free.

This explicit sub-domain was obtained with not too much effort. Let us turn now to the more difficult task to obtain an explicit parametrization for the whole domain.

Uniqueness of the critical point of $\tilde{f}$ for the Vanishing SVI Let us study $\tilde{\phi}(x ; b, \mu)=$ $-\frac{J_{1}(x ; b, \mu)}{j_{2}(x ; b)}$. We want to prove that for each $b \in[0,1]$ and $\left.\mu \in\right]-\infty, \sqrt{3(1-b)}[$, there exists a unique value $x^{*}:=x^{*}(b, \mu) \in\left[\frac{1}{2}, 1\right]$ such that $\inf _{x \in] \frac{1}{2}, 1[} \tilde{\phi}(x)=\tilde{\phi}\left(x^{*}\right)$.

The existence is obvious. The derivative of $\tilde{\phi}$ is

$$
\begin{aligned}
\tilde{\phi}^{\prime}(x ; b, \mu)= & {\left[4 \mu^{2}\left(1-x^{2}\right)\left(2 x^{2}-2 x-1\right)-8 \mu(1-x) \sqrt{1-x^{2}}\left(2 x^{2}-8 x-1\right)+\right.} \\
& \left.+2 x^{4}\left(4-b^{2}\right)-6 x^{3}\left(b^{2}+12\right)+3 x^{2}\left(52-b^{2}\right)+4 x\left(b^{2}-22\right)+3 b^{2}\right] \\
& /\left[8\left(1-x^{2}\right)^{\frac{3}{2}}(1+x)(2 x-1)^{2}\right] .
\end{aligned}
$$

The denominator of the above formula is always positive. Looking at the numerator, for $b=1$ every coefficient of $\mu$ is negative when $x \in] \frac{1}{2}, 1[$ and $\mu<0$, required from the Fukasawa condition. Then $\tilde{\phi}^{\prime}(x ; 1, \mu)$ is always negative and the inferior point of $\tilde{\phi}$ is reached at 1 for every $\mu<0$, so that $x^{*}(1, \mu)=1$. From now on we consider $b<1$. Since for finite values of $\mu, \tilde{\phi}\left(\frac{1}{2} ; b, \mu\right)=\tilde{\phi}(1 ; b, \mu)=\infty$, the points which attain the infimum of $\tilde{\phi}$ are points of minimum belonging to $] \frac{1}{2}, 1[$ and such that $\tilde{\phi}^{\prime}\left(x^{*} ; b, \mu\right)=0$.

What happens when $\mu=-\infty$ ? Dividing by $\mu^{2}$ we still have $\frac{\tilde{\phi}^{\prime}\left(x^{*} ; b, \mu\right)}{\mu^{2}}=0$, so making $\mu$ going to $-\infty$ and relying on the linearity of the limits, we obtain the equation $4\left(1-x^{* 2}\right)\left(2 x^{* 2}-2 x^{*}-1\right)=0$, whose only solution in $\left[\frac{1}{2}, 1\right]$ is 1 , so $x^{*}(b,-\infty)=1$.

Take now $\mu \leq 0$ and $|\nu|<-\mu$ (note in particular that $\mu<\nu$ ) and consider the quantity $\tilde{\phi}(x ; b, \mu)-\tilde{\phi}(x ; b, \nu)$. Unless a factor $b^{-1}(1+x)^{-1}(2 x-1)^{-1}$, it is equal to $(\nu-\mu)(2-x-$ $\left.\frac{(\mu+\nu)}{2} \sqrt{1-x^{2}}\right)$ and, as a consequence, the quantity $\tilde{\phi}^{\prime}(x ; b, \mu)-\tilde{\phi}^{\prime}(x ; b, \nu)$ times $(1+x)(2 x-1)$ equals

$$
(\nu-\mu)\left(-1+\frac{(\mu+\nu)}{2} \frac{x}{\sqrt{1-x^{2}}}\right)+(4 x+1)(\tilde{\phi}(x ; b, \nu)-\tilde{\phi}(x ; b, \mu)) .
$$

The function $\tilde{\phi}$ is decreasing in $\mu$, indeed $\partial_{\mu} \tilde{\phi}(x)=-\frac{2-x-\mu \sqrt{1-x^{2}}}{b(1+x)(2 x-1)}$ and $2-x-\mu \sqrt{1-x^{2}}>2-x-$ $\sqrt{3} \sqrt{1-x^{2}} \geq 0$. So given the fact that $\tilde{\phi}$ is decreasing in $\mu$, we get in turn $\tilde{\phi}^{\prime}(x ; b, \mu)-\tilde{\phi}^{\prime}(x ; b, \nu)<0$. This entails that if there is uniqueness, $x^{*}(b, \nu)<x^{*}(b, \mu)$ under those conditions.

We prove that indeed the uniqueness holds. We prove that if $\tilde{\phi}^{\prime} \geq 0$, then $\tilde{\phi}^{\prime \prime}>0$, which means that once $\tilde{\phi}^{\prime}$ becomes zero, then it will necessarily increase and it can never become 0 again (note that this property is slightly weaker than the convexity of $\tilde{\phi}$ ). We have $\tilde{\phi}^{\prime}=\frac{J_{1} j_{2}^{\prime}-J_{1}^{\prime} j_{2}}{j_{2}^{2}} \geq 0$ but the term $-J_{1}^{\prime} j_{2}$ is negative, so $j_{2}^{\prime}$ must be positive. It holds $\tilde{\phi}^{\prime \prime}=\frac{J_{1} j_{2}^{\prime \prime}-J_{1}^{\prime \prime} j_{2}}{j_{2}^{2}}-2 \tilde{\phi}^{\prime} \frac{j_{2}^{\prime}}{j_{2}}$, where we have proven $-2 \tilde{\phi}^{\prime} \frac{j_{2}^{\prime}}{j_{2}} \geq 0$. We now look at the quantity $n=J_{1} j_{2}^{\prime \prime}-J_{1}^{\prime \prime} j_{2}$ and show that it is strictly positive to obtain the conclusion. Indeed, the denominator of $n$ is $32 \sqrt{1-x^{2}}(1-$ 
$x$ ) which is positive while its numerator is a quadratic function of $\mu$ with quadratic coefficient $-4\left(1-x^{2}\right)(2 x-1)\left(4 x^{2}-2 x-3\right)$, linear coefficient $16 \sqrt{1-x^{2}}(1-x)(2 x-1)\left(2 x^{2}-5 x-4\right)$ and free term depending on $b$. The quadratic coefficient is positive, so $n$ is a convex parabola as a function of $\mu$. Furthermore, the linear coefficient, corresponding to $\left.\partial_{\mu} n(x ; \mu)\right|_{\mu=0}$ unless a positive factor, is negative, so $n(x ; 0)<n(x ; \mu)$ for every $\mu<0$. Then for negative $\mu$ 's it is enough to prove that $n(x ; 0)$ is positive for every $x$. We already know that $j_{2}^{\prime \prime}$ is positive. In addition, $J_{1}^{\prime \prime}=J_{1+}^{\prime \prime} J_{1-}+2 J_{1+}^{\prime} J_{1-}^{\prime}+J_{1+} J_{1-}^{\prime \prime}$ where $J_{1 \pm}^{\prime \prime}(x ; 0)=\left.\frac{\mu}{2\left(1-x^{2}\right)^{\frac{3}{2}}}\right|_{\mu=0}=0$ and $J_{1 \pm}^{\prime}(x ; 0)<0$. Then $J_{1}^{\prime \prime}(x ; 0)>0$ and $n(x ; 0)=J_{1}(x ; 0) j_{2}^{\prime \prime}(x ; 0)-J_{1}^{\prime \prime}(x ; 0) j_{2}(x ; 0)>0$.

For the final case $\mu>0$, we need to introduce the $\mu^{*}$-function.

The $\mu^{*}$ approach for the Vanishing SVI Assume $b<1$ so that $J_{1}(1)>0$ and let us make $\mu$ vary towards its upper bound defined by the Fukasawa condition. Then the function $J_{1}$ will eventually reach the $\mathrm{x}$-axis level at a point $x_{+}^{*}(b)$. This point is necessarily a critical point of $J_{1}$ with $\mu$ set at the value of the upper bound. So $J_{1}\left(x_{+}^{*}(b)\right)=J_{1}^{\prime}\left(x_{+}^{*}(b)\right)=0$. Now observe that $\tilde{\phi}^{\prime}=\frac{J_{1} j_{2}^{\prime}-J_{1}^{\prime} j_{2}}{j_{2}^{2}}$ so that $\tilde{\phi}^{\prime}\left(x_{+}^{*}(b) ; b, \sqrt{3(1-b)}\right)=0$ also. In particular, if we know that $\tilde{\phi}$ (for this critical value of $\mu$ ) has a single critical point, this must be $x_{+}^{*}(b)$.

We look for the solutions to $J_{1}(x)=0$ when $\mu=\sqrt{3(1-b)}$. $J_{1-}$ is always positive on $] \frac{1}{2}, 1[$ while $J_{1+}(x)=1-\frac{x}{2}-\frac{\mu}{2} \sqrt{1-x^{2}}-\frac{b}{4}(1+x)$ satisfies $\left(\left(1-\frac{b}{4}\right) x-\left(\frac{1}{2}+\frac{b}{4}\right)\right)^{2}=0$. This yields

$$
x_{+}^{*}(b)=\frac{\frac{1}{2}+\frac{b}{4}}{1-\frac{b}{4}}=\frac{2+b}{4-b}
$$

which is clearly larger than $\frac{1}{2}$ for positive $b$ and smaller than 1 when $b<1$.

As a sanity check we should verify that also $J_{1}^{\prime}(x)=0$ at this point. Indeed, $J_{1}^{\prime}\left(x_{+}^{*}(b)\right)=$ $J_{1+}^{\prime}\left(x_{+}^{*}(b)\right) J_{1-}\left(x_{+}^{*}(b)\right)+J_{1+}\left(x_{+}^{*}(b)\right) J_{1-}^{\prime}\left(x_{+}^{*}(b)\right)=J_{1+}^{\prime}\left(x_{+}^{*}(b)\right) J_{1-}\left(x_{+}^{*}(b)\right)$. From eq. (8) we have that $J_{1+}^{\prime}$ is null for $\mu=\sqrt{3(1-b)}$ and $x=x_{+}^{*}(b)$.

In the limiting case $b=1$ and $\mu=0$, we have already seen that $J_{1}$ is decreasing and attains its minimum at 1 . Furthermore, $\tilde{\phi}(1 ; 1,0)=0$, so also $\tilde{\phi}$ reaches its minimum at 1 . We can then set $x^{*}(1)=1$. The uniqueness of $x^{*}$ follows from the fact that $J_{1}$ and so $\tilde{\phi}$ are positive in $\left[\frac{1}{2}, 1[\right.$.

Take $x \in] x_{+}^{*}(b), 1\left[\right.$. From eq. (9) we can see that $\tilde{\phi}^{\prime}$ is a concave parabola as a function of $\mu$. So in order to have a unique $\mu=\mu^{*}(x)$-solution to $\tilde{\phi}^{\prime}(x ; b, \mu)=0$, it is enough to prove $\tilde{\phi}^{\prime}(x ; b, \sqrt{3(1-b)})>0$. Note that $x_{+}^{*}(b)$ is a zero and a point of minimum for $\tilde{\phi}(\cdot ; b, \sqrt{3(1-b)})$, so in a right neighborhood of $x_{+}^{*}(b)$ we have $\tilde{\phi}^{\prime}(x ; b, \sqrt{3(1-b)})>0$. If for a certain $x$ we rather have $\tilde{\phi}^{\prime}(x ; b, \sqrt{3(1-b)})<0$, then there exists a $x_{-}^{*}(b)>x_{+}^{*}(b)$ point of maximum such that $\tilde{\phi}^{\prime}\left(x_{-}^{*}(b) ; b, \sqrt{3(1-b)}\right)=0$ and $\tilde{\phi}^{\prime \prime}\left(x_{-}^{*}(b) ; b, \sqrt{3(1-b)}\right)<0$. We show that this is not possible.

Let us look at $J_{1}^{\prime}$. From eq. $\left[8\right.$, we see that $J_{1+}^{\prime}(x)$ is positive iff $\frac{x}{\sqrt{1-x^{2}}}>\frac{b+2}{2 \mu}$ or $x>$ $\frac{2+b}{\sqrt{4 \mu^{2}+(2+b)^{2}}}$. For $\mu=\sqrt{3(1-b)}$ we obtain exactly that $x$ has to be greater than $x_{+}^{*}(b)$. On the other side, $J_{1-}^{\prime}(x)$ is positive iff $x>\frac{2-b}{\sqrt{4 \mu^{2}+(2-b)^{2}}}$ which corresponds to $x>\frac{2-b}{\sqrt{b^{2}-16 b+16}}$ for $\mu=\sqrt{3(1-b)}$. This quantity is inferior to $x_{+}^{*}(b)$ so also $J_{1-}^{\prime}(x)$ is positive. Then $J_{1}^{\prime}\left(x_{-}^{*}(b)\right)>0$. Suppose $x_{-}^{*}(b) \geq x_{m_{2}}$, then $j_{2}^{\prime}\left(x_{-}^{*}(b)\right) \geq 0$ and consequently $\tilde{\phi}^{\prime}\left(x_{-}^{*}(b)\right)>0$, which is not possible. So if it exists, $x_{-}^{*}(b)$ must be smaller than $x_{m_{2}}$. 
We have $\tilde{\phi}^{\prime \prime}=\frac{J_{1} j_{2}^{\prime \prime}-J_{1}^{\prime \prime} j_{2}}{j_{2}^{2}}-2 \tilde{\phi}^{\prime} \frac{j_{2}^{\prime}}{j_{2}}$ so when evaluating in $x_{-}^{*}(b)$, the second term is null. The quantities $J_{1}\left(x_{-}^{*}(b)\right),-j_{2}\left(x_{-}^{*}(b)\right)$ and $j_{2}^{\prime \prime}\left(x_{-}^{*}(b)\right)$ are strictly positive. Also, $J_{1}^{\prime \prime}=J_{1+}^{\prime \prime} J_{1-}+2 J_{1+}^{\prime} J_{1-}^{\prime}+$ $J_{1+} J_{1-}^{\prime \prime}$ and $J_{1 \pm}^{\prime \prime}(x)=\frac{\mu}{2\left(1-x^{2}\right)^{\frac{3}{2}}}$ which are positive. Since also $J_{1 \pm}$ and $J_{1 \pm}^{\prime}$ are positive in $x_{-}^{*}(b)$, then $J_{1}^{\prime \prime}\left(x_{-}^{*}(b)\right)>0$. So $\tilde{\phi}^{\prime \prime}\left(x_{-}^{*}(b) ; b, \sqrt{3(1-b)}\right)$ cannot be negative and this leads to a contradiction.

Consequently, for fixed $b \in] 0,1\left[\right.$, there is a unique function $x \rightarrow \mu^{*}(x)$ such that $\tilde{\phi}^{\prime}\left(x ; b, \mu^{*}(x)\right)=$ 0 is well defined for $x \in] x_{+}^{*}(b), 1[$ and takes values in ] $-\infty, \sqrt{3(1-b)}$ [. It is also continuous since it is defined as the first root of a second degree polynomial with continuously changing parameters. In particular, $\mu^{*}$ is defined as in eq. (5).

Note that the function $\mu^{*}$ cannot be extended to $b=1$ since the inferior point of $\tilde{\phi}$ is reached at 1 for every $\mu<0$. On the other hand, it can be extended to $b=0$ since all the previous statements still hold.

We have already seen that for $x$ in the preimage of $]-\infty, 0]$, the function $\mu^{*}(x)$ is injective. We show that this still holds in the preimage of $] 0, \sqrt{3(1-b)}[$. If this is not the case, there exists a critical point $\hat{x}$ such that $\mu^{* \prime}(\hat{x})=0$. Taking the derivative with respect to $x$ at the members of $\tilde{\phi}^{\prime}\left(x ; \mu^{*}(x)\right)=0$, we obtain $\tilde{\phi}^{\prime \prime}\left(x ; \mu^{*}(x)\right)+\partial_{\mu} \tilde{\phi}^{\prime}\left(x ; \mu^{*}(x)\right) \mu^{* \prime}(x)=0$, so at $\hat{x}$ it holds simultaneously $\tilde{\phi}^{\prime}\left(\hat{x} ; \mu^{*}(\hat{x})\right)=0$ and $\tilde{\phi}^{\prime \prime}\left(\hat{x} ; \mu^{*}(\hat{x})\right)=0$. We want to prove that this is not possible.

At $\hat{x}$, we have $\left.\tilde{\phi}^{\prime \prime}\right|_{\mu=\mu^{*}(\hat{x})}=\left.\frac{J_{1} j_{2}^{\prime \prime}-J_{1}^{\prime \prime} j_{2}}{j_{2}^{2}}\right|_{\mu=\mu^{*}(\hat{x})}$. Since we have already proven that $j_{2}^{\prime \prime}>0$ on ]$\frac{1}{2}, 1\left[\right.$, it is enough to prove that $J_{1}^{\prime \prime}>0$. We consider $\mu \geq 0$. It can be shown that $J_{1}^{\prime \prime}(x)=$ $\frac{\left(4-b^{2}-4 \mu^{2}\right)\left(1-x^{2}\right)^{2}+4 \mu \sqrt{1-x^{2}}\left(2 x^{3}-3 x+2\right)}{8\left(1-x^{2}\right)^{2}}$ and $J_{1}^{\prime \prime \prime}(x)=\frac{3 \mu(2 x-1)}{2\left(1-x^{2}\right)^{\frac{5}{2}}}$, which is positive iff $x>\frac{1}{2}$. So $J_{1}^{\prime \prime}$ is increasing. We have $J_{1}^{\prime \prime}\left(\frac{1}{2}\right)=-\frac{b^{2}}{8}-\frac{\mu^{2}}{2}+\frac{\mu}{\sqrt{3}}+\frac{1}{2}$ which is positive iff $-4 \mu^{2}+\frac{8}{\sqrt{3}} \mu+4-b^{2}>0$; this concave parabola is positive at zero and its positive root is $\frac{2+\sqrt{16-3 b^{2}}}{2 \sqrt{3}}$. This quantity is greater than $\sqrt{3(1-b)}$ so $J_{1}^{\prime \prime}$ is always positive for $\mu \in[0, \sqrt{3(1-b)}[$.

The proof that the function $\mu^{*}(x)$ is injective corresponds to the proof of the uniqueness of $x^{*}(b, \mu)$ in the set $] x_{+}^{*}(b), 1[$. In order to obtain the uniqueness in the whole $] \frac{1}{2}, 1[$, we prove now that $\tilde{\phi}$ is strictly decreasing in $] \frac{1}{2}, x_{+}^{*}(b)\left[\right.$, so that any critical point $x^{*}$ cannot live in this set. For the continuity of $\mu^{*}$ and since $\mu^{*}(1)=-\infty$, for every $\mu \leq 0$ there exists $x>x_{+}^{*}(b)$ such that $\mu^{*}(x)=\mu$. Furthermore, for any fixed $x$, we have proven that there is at most one possible $\mu \leq 0$ such that $\tilde{\phi}^{\prime}(x ; b, \mu)=0$. So if $x<x_{+}^{*}(b)$, there is no $\mu \leq 0$ satisfying the latter equation.

Consider now $\mu>0$. Firstly, we prove that $\tilde{\phi}^{\prime}$ is increasing with respect to $\mu$. From eq. (9) it is evident that it holds true iff $\mu<\frac{(1-x)\left(2 x^{2}-8 x-1\right)}{\sqrt{1-x^{2}}\left(2 x^{2}-2 x-1\right)}$. The right hand side is a decreasing function for $x \in] \frac{1}{2}, 1\left[\right.$ so it is enough to check the inequality in $x_{+}^{*}(b)$. Here the condition becomes $\mu<\frac{b^{2}-8}{b^{2}+4 b-8} \sqrt{3(1-b)}$ and this is greater than $\sqrt{3(1-b)}$, so the inequality holds true.

So in order to prove that $\tilde{\phi}^{\prime}(x ; b, \mu)$ is negative, it is enough to prove that $\tilde{\phi}^{\prime}(x ; b, \sqrt{3(1-b)})$ is non-positive. Suppose now that there exists $x<x_{+}^{*}(b)$ such that $\tilde{\phi}^{\prime}(x ; b, \sqrt{3(1-b)})>0$. Since $\tilde{\phi}^{\prime}\left(\frac{1}{2} ; b, \sqrt{3(1-b)}\right)$ is negative, there is an intermediate point at which $\tilde{\phi}^{\prime}$ is null and then becomes positive up to $x$. Also $\tilde{\phi}^{\prime}\left(x_{+}^{*}(b) ; b, \sqrt{3(1-b)}\right)$ is null so there is $x_{-}^{*}<x_{+}^{*}(b)$ such that $\tilde{\phi}^{\prime}\left(x_{-}^{*} ; b, \sqrt{3(1-b)}\right)>0$ and $\tilde{\phi}^{\prime \prime}\left(x_{-}^{*} ; b, \sqrt{3(1-b)}\right)=0$. However it holds that $\tilde{\phi}^{\prime \prime}\left(x_{-}^{*} ; b, \sqrt{3(1-b)}\right)=$ $\frac{J_{1}\left(x_{-}^{*}\right) j_{2}^{\prime \prime}\left(x_{-}^{*}\right)-J_{1}^{\prime \prime}\left(x_{-}^{*}\right) j_{2}\left(x_{-}^{*}\right)}{j_{2}\left(x_{-}^{*}\right)^{2}}-2 \tilde{\phi}^{\prime}\left(x_{-}^{*}\right) \frac{j_{2}^{\prime}\left(x_{-}^{*}\right)}{j_{2}\left(x_{-}^{*}\right)}$. We have already proven that $J_{1}^{\prime \prime}$ is positive for all $x \in] \frac{1}{2}, 1\left[\right.$ so the first term is positive in $x_{-}^{*}<x_{+}^{*}(b)$. Then, it must be $\tilde{\phi}^{\prime}\left(x_{-}^{*}\right) \frac{j_{2}^{\prime}\left(x_{-}^{*}\right)}{j_{2}\left(x_{-}^{*}\right)}>0$ or equivalently $j_{2}^{\prime}\left(x_{-}^{*}\right)<0$. Note that $J_{1}^{\prime}\left(x_{-}^{*}\right)$ is negative because $J_{1}^{\prime \prime}$ is positive in $] \frac{1}{2}, 1[$ and for 


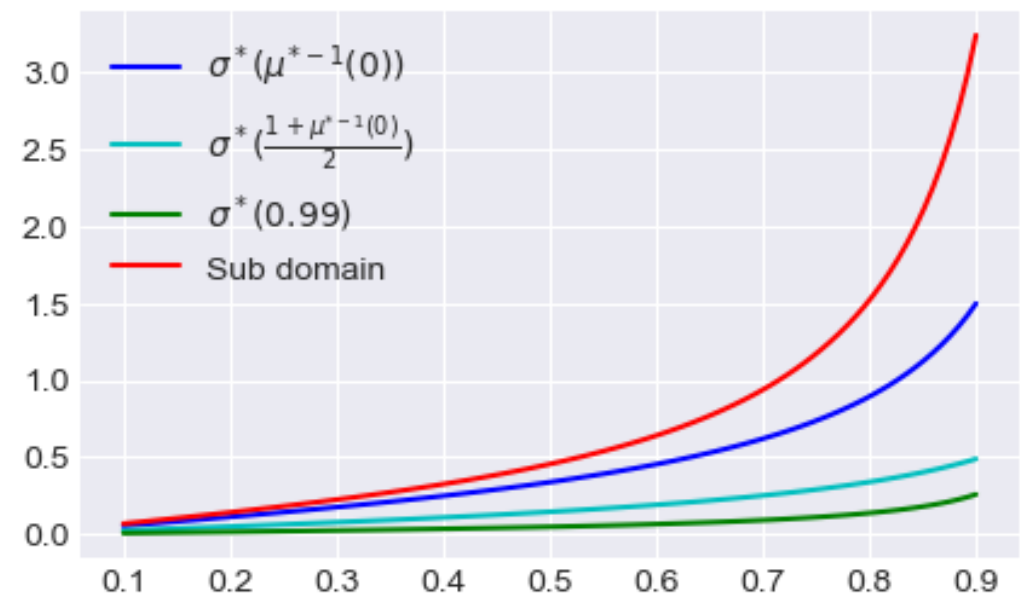

Figure 2: Comparison between the Vanishing Upward SVI arbitrage-free sub-domain and domain as functions of $b$.

$\mu=\sqrt{3(1-b)}$ the function $J_{1}$ is null at $x_{+}^{*}(b)$. Then $\tilde{\phi}^{\prime}\left(x_{-}^{*}\right)=\frac{J_{1}\left(x_{-}^{*}\right) j_{2}^{\prime}\left(x_{-}^{*}\right)-J_{1}^{\prime}\left(x_{-}^{*}\right) j_{2}\left(x_{-}^{*}\right)}{j_{2}\left(x_{-}^{*}\right)^{2}}$ is negative, which is a contradiction.

To sum up, we have shown that for fixed $b \in[0,1[$ and $\mu<\sqrt{3(1-b)}$, there is only one $x$ such that $\tilde{\phi}^{\prime}(x ; b, \mu)=0$ and this $x$ lives in $] x_{+}^{*}(b), 1\left[\right.$. Furthermore, for fixed $b \in\left[0,1[\right.$ and $x \in] x_{+}^{*}(b), 1[$, there is only one $\mu$ in $]-\infty, \sqrt{3(1-b)}\left[\right.$ such that $\tilde{\phi}^{\prime}(x ; b, \mu)=0$. If $b=1$ and $\mu<0$, the function $\tilde{\phi}(x ; 1, \mu)$ is decreasing and reaches its infimum $-\frac{\mu}{2}$ at $x=1$.

\subsubsection{Numerical illustration of Proposition 3.2}

Figure 2 compares the no arbitrage sub-domain for $\sigma$ reported in Lemma 3.3 with the full no arbitrage domain found in Proposition 3.2 , as functions of $b$. The red line represents the subdomain. The blue line corresponds to the full domain for $x^{*}$ equal to the zero of the $\mu^{*}$-function. The reason why we take this point, which depends on $b$, is that the sub-domain is defined for $\mu \leq 0$, so $x^{*}$ must be greater than the zero of $\mu^{*}(\cdot)$. Then, the green line shows the full domain for $x^{*}=0.99$ (for the chosen values of $b, 0.99$ is always greater than the zero of $\mu^{*}(\cdot)$ ) and the light blue line is the full domain for an intermediate point between the blue and the green ones. As expected, the red line is above all the other lines.

\subsection{Vanishing (Downward) SVI}

The Vanishing Downward SVI is the sub-SVI obtained by setting $\rho=-1$ and $a=0$. The corresponding SVI formula becomes

$$
S V I(k ; 0, b,-1, m, \sigma)=b\left(-k+m+\sqrt{(k-m)^{2}+\sigma^{2}}\right) .
$$

We plot in Figure 3 a Vanishing Downward SVI with $b=\frac{1}{2}, m=1$ and $\sigma=1$. 


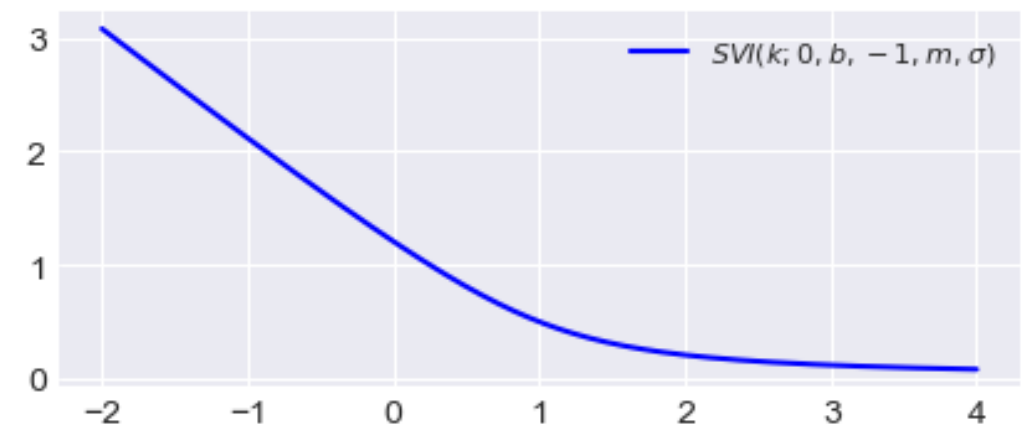

Figure 3: Vanishing Downward SVI with $b=\frac{1}{2}, m=1$ and $\sigma=1$.

From Proposition 2.6, we know that the Vanishing Downward SVI is arbitrage-free iff $S V I(k ; 0, b, 1,-m, \sigma)$ is arbitrage-free, and this corresponds to a Vanishing Upward SVI. This means that the previous results still hold for the Vanishing Downward SVI setting $m(\rho=-1)=-m(\rho=1)$. In particular, we redefine the quantity $\sigma^{*}$ as

$$
\sigma^{*}(x):=-\frac{4 b \sqrt{1-x^{2}}\left(1-x-2 x^{2}\right)}{4\left(2-x+\mu^{*}(x) \sqrt{1-x^{2}}\right)^{2}-b^{2}(1+x)^{2}}
$$

and Proposition 3.2 becomes:

Proposition 3.4 (Fully explicit no arbitrage domain for the Vanishing Downward SVI). A Vanishing Downward SVI with $b=1$ is arbitrage-free iff $\mu>0$ and $\sigma \geq \frac{\mu}{2}$.

$A$ Vanishing Downward SVI with $0<b<1$ is arbitrage-free iff it can be parametrized as

$$
S V I(k)=b \sigma\left(-\frac{k}{\sigma}-\mu^{*}(x)+\sqrt{\left(\frac{k}{\sigma}+\mu^{*}(x)\right)^{2}+1}\right)
$$

where $\frac{2+b}{4-b}<x<1$ and $\sigma \geq \sigma^{*}(x)$.

\section{Extremal Decorrelated SVI}

The coefficient $\rho$ in SVI should correspond to the leverage factor in stochastic volatility models, i.e. the stock/vol returns correlation. In particular, when it is zero, the volatility is independent from the stock which leads to symmetric smiles. In terms of SVI parameters this means that $m$ should also be zero.

This is not automatically enforced in SVI, where $\rho$ and $m$ are distinct parameters. Therefore we call:

- Decorrelated SVI sets of SVI parameters where $\rho=0$;

- Extremal Decorrelated SVI a Decorrelated SVI with $b=2$; 


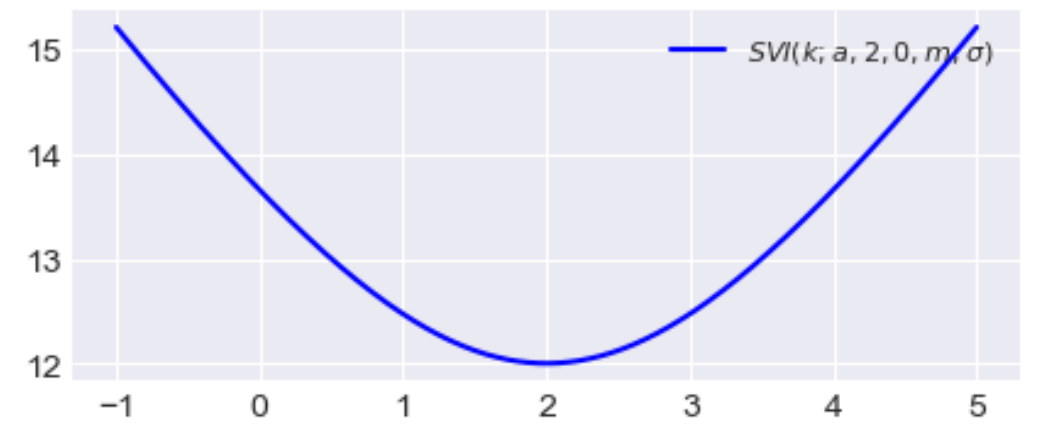

Figure 4: Extremal Decorrelated SVI with $a=8, m=2$ and $\sigma=2$.

- Symmetric SVI a Decorrelated SVI with $m=0$.

The two latter families intersect into an SVI with $b=2$ and $\rho=m=0$.

The Extremal Decorrelated SVI is the sub-SVI obtained by setting $b=2$ and $\rho=0$. The corresponding SVI formula becomes

$$
S V I(k ; a, 2,0, m, \sigma)=a+2 \sqrt{(k-m)^{2}+\sigma^{2}} .
$$

With our notations, $N(l)=\gamma+\sqrt{l^{2}+1}$.

We plot in Figure 4 an Extremal Decorrelated SVI with $a=8, m=2$ and $\sigma=2$.

\subsection{The Fukasawa conditions}

The Roger Lee conditions on $b$ and $\rho$ are satisfied. In subsection 5.2.5 of [6] we prove that the Fukasawa conditions are satisfied iff $\gamma>\tilde{F}(2,0)=0$ and $\left.\mu \in I_{\gamma, 2,0}=\right]-\gamma, \gamma[$. Setting $\mu=q \gamma$ we obtain the following.

Lemma 4.1 (Fukasawa conditions for the Extremal Decorrelated SVI). An Extremal Decorrelated SVI satisfies the Fukasawa conditions iff it can be parametrized by

$$
2 \sigma\left(\gamma+\sqrt{\left(\frac{k}{\sigma}-q \gamma\right)^{2}+1}\right)
$$

with $\gamma>0, q \in]-1,1[$ and $\sigma>0$.

\subsection{The condition on $\sigma$}

We will prove the following characterization of the no Butterfly arbitrage domain for the Extremal Decorrelated SVI:

Proposition 4.2 (Fully explicit no arbitrage domain for the Extremal Decorrelated SVI). An Extremal Decorrelated SVI is arbitrage-free iff it can be parametrized as

$$
S V I(k)=2 \sigma\left(\gamma+\sqrt{\left(\frac{k}{\sigma}-q \gamma\right)^{2}+1}\right)
$$

with $\gamma>0, q \in]-1,1\left[\right.$ and $\sigma \geq \frac{1}{\gamma(1-|q|)}$. 


\subsubsection{Proof of Proposition 4.2}

Setting $\mu=q \gamma$ with $q \in]-1,1\left[\right.$, the function $G_{1}$ is

$$
G_{1}(l)=\frac{-2 q \gamma l^{3}+l^{2}\left(\left(3+q^{2}\right) \gamma^{2}+3\right)-4 q \gamma l+4\left(1+\gamma^{2}\right)+2 \gamma \sqrt{l^{2}+1}\left(l^{2}-2 q \gamma l+4\right)}{4\left(l^{2}+1\right)\left(\gamma+\sqrt{l^{2}+1}\right)^{2}}
$$

and since $\rho=0, g_{2}(l)=-\frac{\left(l^{2}-2\right) \sqrt{l^{2}+1}-2 \gamma}{2\left(l^{2}+1\right)^{\frac{3}{2}}\left(\gamma+\sqrt{l^{2}+1}\right)}$ is symmetric, so $l_{1}=-l_{2}$. In particular, working with $l \geq 0$, to find its roots it is sufficient to solve a third degree equation in $\sqrt{l^{2}+1}$.

We operate the substitution $y=\sqrt{l^{2}+1}$ so that

$$
\begin{aligned}
G_{1}(l(y)) & =\frac{2 \gamma y^{3}+\left(\left(3+q^{2}\right) \gamma^{2}+3\right) y^{2}+6 \gamma y+1+\left(1-q^{2}\right) \gamma^{2}-2 q \gamma l(y)\left(y^{2}+2 \gamma y+1\right)}{4 y^{2}(y+\gamma)^{2}}, \\
g_{2}(l(y)) & =-\frac{y^{3}-3 y-2 \gamma}{2 y^{3}(y+\gamma)} .
\end{aligned}
$$

From Lemma 2.5. it follows that the function $f=-\frac{b g_{2}}{2 G_{1}}$ is such that for $l>l_{2}$ and $q$ negative, $f(l)<f(-l)$ while for $q$ positive, $f(l)>f(-l)$ and for $q=0$ it is symmetric. So if we want to find the supremum of $f$ in $]-\infty, l_{1}[\cup] l_{2}, \infty[$, it is enough to look at $]-\infty, l_{1}[$ for $q<0$ and $] l_{2}, \infty[$ for $q \geq 0$. Note also that $f(l ; \gamma, q)=f(-l ; \gamma,-q)$.

Suppose $q \geq 0$ and $l>l_{2}$. The function $f$ has its limit at $\infty$ equal to $\frac{1}{\gamma(1-q)}$. We want to prove that this is also the supremum of $f$. This would be true iff $f(l)<\frac{1}{\gamma(1-q)}$ for every $l$ or equivalently iff $-g_{2}(l) \gamma(1-q)<G_{1}(l)$. Using the change of variable, this reads also

$$
\frac{y^{3}-3 y-2 \gamma}{y^{3}(y+\gamma)} \gamma(1-q)<\frac{2 \gamma y^{3}+\left(\left(3+q^{2}\right) \gamma^{2}+3\right) y^{2}+6 \gamma y+1+\left(1-q^{2}\right) \gamma^{2}-2 q \gamma \sqrt{y^{2}-1}\left(y^{2}+2 \gamma y+1\right)}{2 y^{2}(y+\gamma)^{2}} \text {. }
$$

Multiplying by $2 y^{3}(y+\gamma)^{2}$ and simplifying, one gets

$$
\begin{aligned}
2 \gamma q y^{4}+\left(\gamma^{2}(1+q)^{2}+3\right) y^{3}+6 \gamma(2-q) y^{2}+\left(\gamma^{2}(1-q)(q+11)+1\right) y+ \\
4 \gamma^{3}(1-q)-2 \gamma q y \sqrt{y^{2}-1}\left(y^{2}+2 \gamma y+1\right)>0 .
\end{aligned}
$$

Since $\sqrt{y^{2}-1}<y$, the LHS is greater than

$$
\left(\gamma^{2}(1-q)^{2}+3\right) y^{3}+4 \gamma(3-2 q) y^{2}+\left(\gamma^{2}(1-q)(q+11)+1\right) y+4 \gamma^{3}(1-q) .
$$

Each of the coefficients of this polynomial is positive and $y>0$ so the whole polynomial is positive and $f$ reaches its supremum at $\infty$.

In the case $q<0$, the conclusion follows immediately from Proposition 2.6 .

\section{$5 \quad$ Symmetric SVI}

The Symmetric SVI is the sub-SVI obtained by setting $\rho=0$ and $m=0$. The corresponding SVI formula becomes

$$
S V I(k ; a, b, 0,0, \sigma)=a+b \sqrt{k^{2}+\sigma^{2}} .
$$

With our notations, $N(l)=\gamma+\sqrt{l^{2}+1}$.

We plot in Figure 5 a Symmetric SVI with $a=0.64, b=1.6$ and $\sigma=0.4$. 


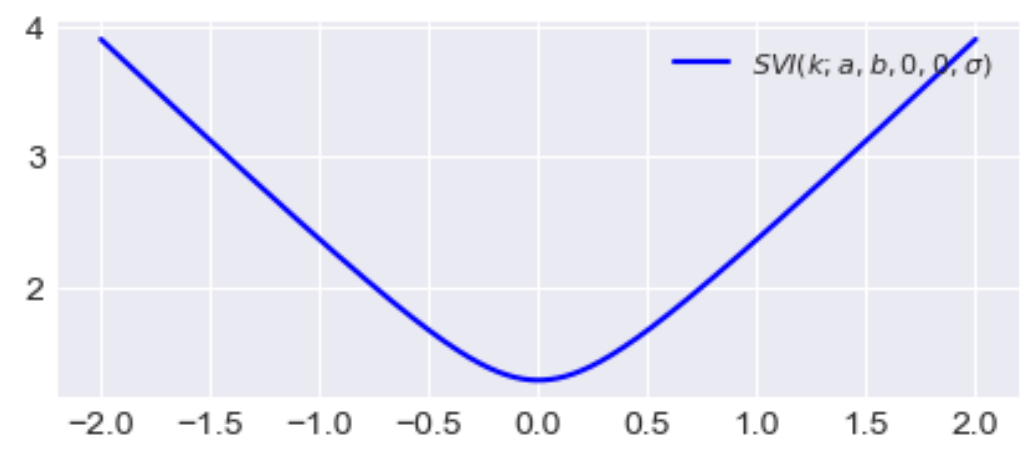

Figure 5: Symmetric SVI with $a=0.64, b=1.6$ and $\sigma=0.4$.

\subsection{The Fukasawa conditions}

The Roger Lee conditions imply $0<b \leq 2$. In Appendix B of [6] we prove that the Fukasawa conditions are satisfied iff $\gamma>\tilde{F}(b, 0)=g_{-(b, 0)}\left(-\frac{6 b}{\sqrt{b^{4}-20 b^{2}+64}}\right)=-\frac{\left(b^{2}+32\right) \sqrt{4-b^{2}}}{\left(16-b^{2}\right)^{\frac{3}{2}}}$. Since $\rho=0$, the interval for $\mu$ is symmetric and being $\mu=0$, the Fukasawa condition on $\mu$ is automatically satisfied.

Lemma 5.1 (Fukasawa conditions for the Symmetric SVI). A Symmetric SVI with $0<b \leq 2$ satisfies the Fukasawa conditions iff $\gamma>\tilde{F}(b)=-\frac{\left(b^{2}+32\right) \sqrt{4-b^{2}}}{\left(16-b^{2}\right)^{\frac{3}{2}}}$.

For every value of $b$, we have $\tilde{F}(b) \in]-1,0]$ and in particular $\tilde{F}(2)=0$. We can extend the definition of $\tilde{F}$ to the point $b=0$, setting $\tilde{F}(0)=-1$. Furthermore, $\frac{d \tilde{F}}{d b}(b)=\frac{108 b^{3}}{\left(16-b^{2}\right)^{\frac{5}{2}} \sqrt{4-b^{2}}}>0$ for positive $b$. So the inverse function $\tilde{F}^{-1}:[-1,0] \rightarrow[0,2]$ is well defined. As in section 2.3 , we define

$$
\tilde{G}(\gamma)= \begin{cases}\tilde{F}^{-1}(\gamma) & \text { if } \gamma \in]-1,0] \\ 2 & \text { if } \gamma>0\end{cases}
$$

It can be shown, working out the calculation of the roots of a 3rd degree polynomial, that the explicit formula of $\tilde{G}$ in the case $\gamma \leq 0$ is

$$
\tilde{G}(\gamma)=2 \sqrt{\frac{6 \sqrt{8 \gamma^{2}+1} \cos \left(\frac{1}{3} \arccos \left(-\frac{8 \gamma^{4}+20 \gamma^{2}-1}{\left(8 \gamma^{2}+1\right)^{\frac{3}{2}}}\right)\right)-4 \gamma^{2}-5}{1-\gamma^{2}}} .
$$

\subsection{The condition on $\sigma$}

Before enunciating the main Proposition of this chapter, we need to introduce some notation. In this section, for positive $l \mathrm{~s}$ we will operate a change of variable setting $z=\frac{1}{\sqrt{l^{2}+1}}$, whose domain is $] 0,1]$. Then we define the functions $J_{1}(z):=G_{1}(l(z)), j_{2}(z):=g_{2}(l(z)), \eta(z):=h(l(z))$ and 
$j(z):=g(l(z))$. In particular,

$$
\begin{aligned}
j_{2}(z ; \gamma) & =z \frac{2 \gamma z^{3}+3 z^{2}-1}{2(\gamma z+1)}, \\
\eta(z ; \gamma) & =1-\frac{1-z^{2}}{2(1+\gamma z)}, \\
j(z) & =\frac{1-z^{2}}{4} .
\end{aligned}
$$

In the following, the symbol ' indicates the derivative with respect to $z$ if not differently specified. We also define the functions

$$
\begin{aligned}
\gamma^{*}(u) & :=u \sqrt{6 u^{3}+15 u^{2}+14 u+6+(1+u)^{2} \sqrt{3\left(12 u^{2}+12 u+11\right)}}, \\
b^{*}(z, \gamma) & :=\sqrt{\frac{\eta(z)\left(\eta(z) j_{2}^{\prime}(z)-2 \eta^{\prime}(z) j_{2}(z)\right)}{j(z)\left(j(z) j_{2}^{\prime}(z)-2 j^{\prime}(z) j_{2}(z)\right)}}, \\
\sigma^{*}(z, \gamma) & :=-\frac{b^{*}\left(z^{*}, \gamma\right) j_{2}\left(z^{*}\right)}{2\left(\eta\left(z^{*}\right)^{2}-b^{*}\left(z^{*}, \gamma\right)^{2} j\left(z^{*}\right)^{2}\right)} .
\end{aligned}
$$

The final result of this subsection will be:

Proposition 5.2 (Fully explicit no arbitrage domain for the Symmetric SVI). An arbitrage-free Symmetric SVI must have $\gamma>-1$.

A Symmetric SVI with $b=2$ is arbitrage-free iff $\gamma>0$ and $\sigma \geq \frac{1}{\gamma}$.

Call $z^{*}\left(\gamma^{*}(u), 0\right)=\frac{u}{\gamma^{*}(u)}$ and $z^{*}(\gamma, \tilde{G}(\gamma))=\frac{\sqrt{\left(4-\tilde{G}(\gamma)^{2}\right)\left(16-\tilde{G}(\gamma)^{2}\right)}}{\tilde{G}(\gamma)^{2}+8}$.

- If $\gamma=-\sqrt{\frac{9+5 \sqrt{3}}{18}}$, the Symmetric SVI is arbitrage-free iff $b<2 \sqrt{3 \sqrt{3}-5}$ and $\sigma \geq-\frac{b j_{2}(\hat{z})}{2\left(\eta(\hat{z})^{2}-b^{2} j(\hat{z})^{2}\right)}$ where $\hat{z}=\sqrt{\frac{3-\sqrt{3}}{2}}$.

- If $\gamma \neq-\sqrt{\frac{9+5 \sqrt{3}}{18}}$ and $b<2$, a Symmetric SVI is arbitrage-free iff it can be parametrized as

$$
S V I(k)=\sigma b^{*}\left(z, \gamma^{*}(u)\right)\left(\gamma^{*}(u)+\sqrt{\left(\frac{k}{\sigma}\right)^{2}+1}\right)
$$

where

$$
\begin{aligned}
& \square u>-1, \\
& \square z \in] z^{*}\left(\gamma^{*}(u), 0\right), z^{*}\left(\gamma^{*}(u), \tilde{G}\left(\gamma^{*}(u)\right)\right)\left[\text { if } \gamma^{*}(u)<-\sqrt{\frac{9+5 \sqrt{3}}{18}}\right. \text { or } \\
& z \in] z^{*}\left(\gamma^{*}(u), \tilde{G}\left(\gamma^{*}(u)\right)\right), z^{*}\left(\gamma^{*}(u), 0\right)\left[\text { if } \gamma^{*}(u)>-\sqrt{\frac{9+5 \sqrt{3}}{18}},\right. \\
& \square \sigma \geq \sigma^{*}\left(z, \gamma^{*}(u)\right) .
\end{aligned}
$$




\subsubsection{Proof of Proposition 5.2}

When $b=2$, we are in the case of the Extremal Decorrelated SVI with $q=0$, so the arbitrage conditions are satisfied iff $\gamma>0$ and $\sigma \geq \frac{1}{\gamma}$.

From now on we consider $b<2$. Since $\rho=0$, the $g_{2}$ function is symmetric with respect to $l$ and since $\mu=0$, the $G_{1}$ function is symmetric too. Then $\tilde{f}=-\frac{G_{1}}{g_{2}}$ is symmetric as a function of $l$ and we consider $l \geq 0$. Recall that we are interested in the value of $\sigma^{*}=\sup _{l>l_{2}} \frac{b}{2 \tilde{f}(l)}$.

The function $\tilde{\phi}(z):=\tilde{f}(l(z))$ goes to $\infty$ at 0 and at the non trivial zero of $j_{2}$, which we call $z_{2}(\gamma)$. Then $\tilde{\phi}$ has at least one point of minimum in $] 0, z_{2}[$.

Study of $j_{2}$ To find $z_{2}$, we should solve $p_{1}(z):=2 \gamma z^{3}+3 z^{2}-1=0$. It can be shown that for $\gamma$ negative, the polynomial has three roots but only the second one lies in the interval $] 0,1[$. If $\gamma$ is null, then $z_{2}(0)=\frac{1}{\sqrt{3}}$. If $\gamma$ is positive but not greater than 1 , the polynomial has three roots and only the third lies in the interval ]0,1[. Finally, if $\gamma>1$, there is only one root. Using the trigonometric notation, $z_{2}(\gamma)$ is

$$
z_{2}(\gamma)= \begin{cases}-\frac{1}{\gamma} \cos \left(\frac{1}{3} \arccos \left(1-2 \gamma^{2}\right)-\frac{2 \pi}{3}\right)-\frac{1}{2 \gamma} & \text { if } \gamma<0 \\ \frac{1}{\sqrt{3}} & \text { if } \gamma=0 \\ \frac{1}{\gamma} \cos \left(\frac{1}{3} \arccos \left(2 \gamma^{2}-1\right)\right)-\frac{1}{2 \gamma} & \text { if } 0<\gamma \leq 1 \\ \frac{1}{\gamma} \cosh \left(\frac{1}{3} \operatorname{arccosh}\left(2 \gamma^{2}-1\right)\right)-\frac{1}{2 \gamma} & \text { if } \gamma>1\end{cases}
$$

Let us show that $j_{2}$ has a single critical point $z_{m_{2}}$ where it achieves its minimum. It holds

$$
\begin{aligned}
j_{2}^{\prime}(z) & =\frac{6 \gamma^{2} z^{4}+14 \gamma z^{3}+9 z^{2}-1}{2(\gamma z+1)^{2}}, \\
j_{2}^{\prime \prime}(z) & =\frac{6 \gamma^{3} z^{4}+19 \gamma^{2} z^{3}+21 \gamma z^{2}+9 z+\gamma}{(\gamma z+1)^{3}} .
\end{aligned}
$$

Since $j_{2}^{\prime}(0)=-\frac{1}{2}$ and $j_{2}^{\prime}(1)=\frac{3 \gamma+4}{\gamma+1}$, which is positive having $\gamma>-1$, then $j_{2}$ has at least one critical point in $] 0,1[$.

- The function $j_{2}^{\prime \prime}$ is positive if $\gamma \geq 0$ so $j_{2}$ has exactly one critical point in this case, which is a point of minimum.

- Consider the case $\gamma<0$. The polynomial $p_{2}(z):=6 \gamma^{2} z^{4}+14 \gamma z^{3}+9 z^{2}-1$ has derivative $p_{2}^{\prime}(z)=2 z\left(12 \gamma^{2} z^{2}+21 \gamma z+9\right)$, so its three critical points are $0,-\frac{3}{4 \gamma}$ and $-\frac{1}{\gamma}$. Since $\gamma>-1$, the third critical point, which is a point of minimum, is greater than 1 and, since 0 is the first critical point and since $-1=p_{2}(0)<0<p_{2}(1)=6 \gamma^{2}+14 \gamma+8=2(\gamma+1)(\gamma+4), p_{2}$ has exactly one zero in $] 0,1\left[\right.$. So again $j_{2}$ has only one critical point in this interval.

Because $j_{2}$ is negative before $z_{2}$ then $j_{2}^{\prime}\left(z_{2}\right)>0$, furthermore $j_{2}^{\prime}(0)<0$ so the critical point $z_{m_{2}}$ of $j_{2}$ (a point of minimum) lies in $] 0, z_{2}[$.

We have already seen that if $\gamma \geq 0$, then $j_{2}$ is strictly convex in $] 0,1[$. 
We can moreover show that $j_{2}$ has a single inflection point if $\gamma$ is negative. In such case, call the numerator of $j_{2}^{\prime \prime}$ as $p_{3}(z):=6 \gamma^{3} z^{4}+19 \gamma^{2} z^{3}+21 \gamma z^{2}+9 z+\gamma$. It holds $p_{3}(0)=\gamma<0$ and $p_{3}(1)=6 \gamma^{3}+19 \gamma^{2}+22 \gamma+9$, which is a third degree polynomial in $\gamma$ with only root equal to -1 and a positive leading order term, so it is positive for $\gamma>-1$. Then $p_{3}$ has at least one zero in ]0,1[. Moreover, $p_{3}^{\prime}(z)=3(\gamma z+1)^{2}(8 \gamma z+3)$, whose zeros are $-\frac{3}{8 \gamma}$ and $-\frac{1}{\gamma}>1$. The former is a point of maximum for $p_{3}$ while the latter is an inflection point. Gathering all the informations, it follows that $p_{3}$ has exactly one zero in ]0,1[. Since $j_{2}^{\prime \prime}>0$ at the point of minimum of $j_{2}$ and $j_{2}^{\prime \prime}(0)<0$, the only inflection point $z_{i_{2}}(\gamma)$ of $j_{2}$ lies in $] 0, z_{m_{2}}[$.

Study of $J_{1}$ It holds $J_{1}(z)=\eta(z)^{2}-b^{2} j(z)^{2}=\left(1-\frac{1-z^{2}}{2(1+\gamma z)}\right)^{2}-b^{2} \frac{1-z^{2}}{16}$.

We write here a general result which holds for every $S V I$.

Lemma 5.3. Under the Fukasawa conditions, $G_{1}$ is strictly decreasing at the second zero of $g_{2}$.

Proof. Since $G_{1}^{\prime}=G_{1+}^{\prime} G_{1-}+G_{1+} G_{1-}^{\prime}$ and $G_{1+}^{\prime}<G_{1-}^{\prime}$ for $l>l^{*}$, then it is enough to prove $G_{1-}^{\prime}\left(l_{2}\right)<0$. Remember that $G_{1-}\left(l_{2}\right)=1-N^{\prime}\left(l_{2}\right)\left(\frac{l_{2}+\mu}{2 N}-\frac{b}{4}\right)$. From the formula of $g_{2}$, we have that $N^{\prime \prime}\left(l_{2}\right)=\frac{N^{\prime}\left(l_{2}\right)^{2}}{2 N\left(l_{2}\right)}$. Then

$$
\begin{aligned}
G_{1-}^{\prime}\left(l_{2}\right) & =-\frac{N^{\prime \prime}\left(l_{2}\right)\left(l_{2}+\mu\right)+N^{\prime}\left(l_{2}\right)}{2 N\left(l_{2}\right)}+\frac{N^{\prime}\left(l_{2}\right)^{2}\left(l_{2}+\mu\right)}{2 N\left(l_{2}\right)^{2}}+\frac{b N^{\prime \prime}\left(l_{2}\right)}{4} \\
& =\frac{N^{\prime}\left(l_{2}\right)^{2}\left(l_{2}+\mu\right)}{4 N\left(l_{2}\right)^{2}}+\frac{b N^{\prime}\left(l_{2}\right)^{2}}{8 N\left(l_{2}\right)}-\frac{N^{\prime}\left(l_{2}\right)}{2 N\left(l_{2}\right)} .
\end{aligned}
$$

For the absence of arbitrage, $\mu<\inf _{l>l^{*}} L_{+}(l)=\inf _{l>l^{*}}\left(\frac{2 N(l)}{N^{\prime}(l)}-\frac{b N(l)}{2}-l\right)$ so this holds in particular in $l_{2}>l^{*}$. Substituting with the formula of $L_{+}$we obtain

$$
G_{1-}^{\prime}\left(l_{2}\right)<\frac{N^{\prime}\left(l_{2}\right)^{2}}{4 N\left(l_{2}\right)^{2}}\left(l_{2}+\frac{2 N\left(l_{2}\right)}{N^{\prime}\left(l_{2}\right)}-\frac{b N\left(l_{2}\right)}{2}-l_{2}\right)+\frac{b N^{\prime}\left(l_{2}\right)^{2}}{8 N\left(l_{2}\right)}-\frac{N^{\prime}\left(l_{2}\right)}{2 N\left(l_{2}\right)}=0 .
$$

Observe that

$$
\begin{aligned}
J_{1}^{\prime}(z) & =\frac{\gamma z^{4}\left(b^{2} \gamma^{2}+4\right)+z^{3}\left(3 b^{2} \gamma^{2}+8 \gamma^{2}+8\right)+3 \gamma z^{2}\left(b^{2}+8\right)+z\left(b^{2}+8 \gamma^{2}+8\right)+4 \gamma}{8(\gamma z+1)^{3}}, \\
J_{1}^{\prime \prime}(z) & =\frac{b^{2}}{8}+\frac{\gamma^{2} z^{4}+4 \gamma z^{3}+6 z^{2}+(4 \gamma z+1)\left(2-\gamma^{2}\right)}{2(\gamma z+1)^{4}}, \\
J_{1}^{\prime \prime \prime}(z) & =\frac{6 z\left(\gamma^{2}-1\right)^{2}}{(\gamma z+1)^{5}} .
\end{aligned}
$$

So:

- For $\gamma \geq 0, J_{1}^{\prime}$ is always greater than 0 so $J_{1}$ is increasing and has its minimum in 0 .

- If $\gamma<0, J_{1}^{\prime}$ is negative for $z=0$ and we know that $G_{1}^{\prime}\left(l_{2}\right)<0$ so $J_{1}^{\prime}\left(z_{2}\right)>0$. Then $J_{1}$ has at least a point of minimum in $] 0, z_{2}$ [. Also, the minimum of $J_{1}^{\prime \prime}$ is reached at 0 and it is $\frac{b^{2}-4 \gamma^{2}+8}{8}$. This quantity is positive iff $\gamma^{2}<\frac{b^{2}+8}{4}$, so for example for every $\gamma<0$ because $\gamma$ is greater than -1 . In such case $J_{1}$ is always convex and it has exactly one critical point $z_{m_{1}}$, which is a point of minimum, in $] 0, z_{2}[$. 
Note also that when $\gamma$ goes to its Fukasawa limit, $J_{1+}(z)=1-\frac{1-z^{2}}{2(\gamma z+1)}-\frac{b \sqrt{1-z^{2}}}{4}$ goes to 0 in $z_{+}^{*}(b):=\frac{\sqrt{\left(4-b^{2}\right)\left(16-b^{2}\right)}}{b^{2}+8}$, which is also a point of minimum for $J_{1}$. It is easy to shown that if $\gamma>-\frac{\left(b^{4}-38 b^{2}+64\right)\left(b^{2}+8\right)}{\left(4-b^{2}\right)^{\frac{3}{2}}\left(16-b^{2}\right)^{\frac{3}{2}}}:=M(b)$, then $\left.j_{2}\left(z_{+}^{*}(b)\right)\right)>0$, so $z_{+}^{*}(b)>z_{2}(\gamma)$, otherwise $z_{+}^{*}(b) \leq z_{2}(\gamma)$. Note that $M(b) \geq \tilde{F}(b)$ (the equality holding iff $b=0$ ), so when $\gamma$ goes to $\tilde{F}(b), J_{1}$ reaches its minimum before $z_{2}(\gamma)$ (or at $z_{2}(\gamma)$ if $b=0$ ).

Then $J_{1}$ has in any case one point of minimum: in the case $\gamma \geq 0$, this is 0 , while when $\gamma<0$, it is $z_{m_{1}}(\gamma, b)$. Also, $J_{1}$ is convex if $\gamma^{2} \leq \frac{b^{2}+8}{4}$ (in particular $\gamma<0$ given that $\gamma$ should be larger than -1$)$ while it has a unique inflection point $z_{i_{1}}(\gamma, b)$ if $\gamma^{2}>\frac{b^{2}+8}{4}$.

Uniqueness of the critical point of $\tilde{\phi}$ for the Symmetric SVI From the formula for $\tilde{\phi}^{\prime}=$ $\frac{J_{1} j_{2}^{\prime}-J_{1}^{\prime} j_{2}}{j_{2}^{2}}$, given that $J_{1}>0$ and $j_{2}<0$ we have that:

- for $\gamma<0$ : all the critical points of $\tilde{\phi}$ must lie in $\left[z_{m_{1}}, z_{m_{2}}\right] \cup\left[z_{m_{2}}, z_{2} \wedge z_{m_{1}}\right)$;

- for $\gamma \geq 0$ : all the critical points of $\tilde{\phi}$ must lie in $] 0, z_{m_{2}}$ [ since $J_{1}^{\prime} j_{2}$ is negative.

Considering now the second derivative of $\tilde{\phi}$, so $\tilde{\phi}^{\prime \prime}=\frac{J_{1} j_{2}^{\prime \prime}-J_{1}^{\prime \prime} j_{2}}{j_{2}^{2}}-2 \tilde{\phi}^{\prime} \frac{j_{2}^{\prime}}{j_{2}}$ and evaluating it at a point of maximum of $\tilde{\phi}$, the second term becomes null and necessarily $\tilde{\phi}^{\prime \prime} \leq 0$. Then it must hold $j_{2}^{\prime \prime} J_{1}^{\prime \prime} \leq 0$ (the equality holding when both factors are 0 ). In particular:

1. for $\gamma<0$ : if $\tilde{\phi}$ has a point of maximum, it lies in $\left[z_{m_{1}}, z_{i_{2}}(\gamma)\right.$ [ because in such case $J_{1}^{\prime \prime}>0$, $j_{2}^{\prime \prime}<0$ in $\left[0, z_{i_{2}}(\gamma)[\right.$

2. for $0 \leq \gamma \leq \sqrt{\frac{b^{2}+8}{4}}$ : $\tilde{\phi}$ cannot have a point of maximum, because $J_{1}$ and $j_{2}$ are strictly convex, so it has one point of minimum which lies in $] 0, z_{m_{2}}[$;

3. for $\gamma>\sqrt{\frac{b^{2}+8}{4}}$ : $J_{1}$ has one inflection point $z_{i_{1}}$ and if $\tilde{\phi}$ has a point of maximum, it lies in ] $0, z_{m_{2}} \wedge z_{i_{1}}[$.

We consider the first case and show that $z_{m_{1}}>z_{i_{2}}(\gamma)$ or equivalently that $J_{1}^{\prime}\left(z_{i_{2}}\right)<0$, so that there is no point of maximum. The quantity $z_{i_{2}}(\gamma)$ does not depend on $b$ so $\partial_{b^{2}}\left(J_{1}^{\prime}\left(z_{i_{2}}\right)\right)=$ $\partial_{b^{2}} J_{1}^{\prime}\left(z_{i_{2}}\right)=\frac{z_{i_{2}}}{8}>0$. We can then check that for every $-1<\gamma<0$ we have indeed $J_{1}^{\prime}\left(z_{i_{2}} ; b=2\right)<0$ in Figure6.

Let us now consider the last case. Since $\gamma>\sqrt{\frac{b^{2}+8}{4}}$, then in particular $\gamma^{2}>2$. Suppose we are at a critical point of $\tilde{\phi}$, then $J_{1}=\frac{J_{1}^{\prime}}{j_{2}^{\prime}} j_{2}$ and its second derivative evaluated at this point is $\tilde{\phi}^{\prime \prime}=\frac{J_{1} j_{2}^{\prime \prime}-J_{1}^{\prime \prime} j_{2}}{j_{2}^{2}}=\frac{J_{1}^{\prime} j_{2}^{\prime \prime}-J_{1}^{\prime \prime} j_{2}^{\prime}}{j_{2} j_{2}^{\prime}}$. In the set $] 0, z_{m_{2}} \wedge z_{i_{1}}[$, the denominator is always positive. The numerator times $16(\gamma z+1)^{6}$ is equal to

$$
\begin{aligned}
& b^{2}\left(6 \gamma^{6} z^{8}+36 \gamma^{5} z^{7}+91 \gamma^{4} z^{6}+126 \gamma^{3} z^{5}+3 \gamma^{2}\left(\gamma^{2}+34\right) z^{4}+2 \gamma\left(5 \gamma^{2}+23\right) z^{3}+3\left(4 \gamma^{2}+3\right) z^{2}+6 \gamma z+1\right)+ \\
& +24 \gamma^{4} z^{8}+96 \gamma^{3}\left(\gamma^{2}+1\right) z^{7}+4 \gamma^{2} z^{6}\left(148 \gamma^{2}+17\right)+24 \gamma z^{5}\left(8 \gamma^{4}+48 \gamma^{2}-3\right)+12 z^{4}\left(50 \gamma^{4}+81 \gamma^{2}-6\right)+ \\
& +16 \gamma z^{3}\left(44 \gamma^{2}+25\right)+12 z^{2}\left(33 \gamma^{2}+8\right)+120 \gamma z+4 \gamma^{2}+8
\end{aligned}
$$




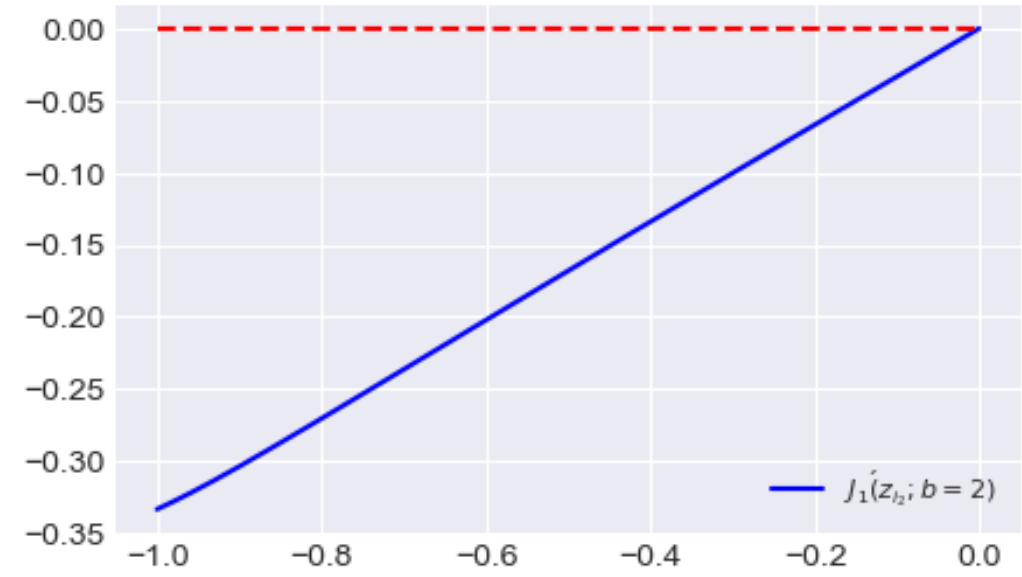

Figure 6: $J_{1}^{\prime}\left(z_{i_{2}} ; b=2\right)$ as a function of $\gamma$.

All coefficients of $z$ are positive quantities, indeed the only minus involved are in the expressions $8 \gamma^{4}+48 \gamma^{2}-3>8 \times 4+48 \times 2-3>0$ and $50 \gamma^{4}+81 \gamma^{2}-6>50 \times 4+81 \times 2-6>0$. Then $\tilde{\phi}^{\prime \prime}$ is positive and $\tilde{\phi}$ cannot have a point of maximum even in this case.

We denote from now on the well-defined single point of minimum of $\tilde{\phi}$ with $z^{*}(\gamma, b)$.

Limits of $z^{*}$ with respect to $\gamma$ When $\gamma$ goes to $\infty$, the ratio between the numerator of $\tilde{\phi}^{\prime}$ and $\gamma^{4}$ goes to $2 z^{6}\left(48+b^{2}\left(z^{2}-3\right)\right)$, which is null for $z=0$, so $z^{*}(\infty, b)=0$.

On the other side, let $\gamma$ go to $\tilde{F}(b)$. Then, from the above study of $J_{1}$, the minimum point of $J_{1}$ is $z_{+}^{*}(b)=\frac{\sqrt{\left(4-b^{2}\right)\left(16-b^{2}\right)}}{b^{2}+8}<z_{2}(\tilde{F}(b))$ and it coincides with the minimum point $z^{*}(\tilde{F}(b), b)$ of $\tilde{\phi}$, at which $\tilde{\phi}$ is null.

If $b=0$, it still holds $z^{*}(\infty, 0)=0$ and it is easy to show that at the point $z^{*}(0)=z_{2}(\tilde{F}(0))=1$ the function $\tilde{\phi}$ with $\gamma=\tilde{F}(b)$ vanishes.

The $b^{*}$ approach for the Symmetric SVI Let $\left.\gamma \in\right]-1, \infty[$ be fixed. With reference to the definition of $\tilde{G}(\gamma)$ in eq. 13 , we want to prove that for every $b \in] 0, \tilde{G}(\gamma)$ [ the corresponding $z^{*}(\gamma, b)$ lies in $] z^{*}(\gamma, 0), z^{*}(\gamma, \tilde{G}(\gamma))[$ (or in $] z^{*}(\gamma, \tilde{G}(\gamma)), z^{*}(\gamma, 0)[)$.

Remark 5.4. Note that if $\gamma>0, b$ could actually attain the value $\tilde{G}(\gamma)=2$ but we have already seen in the Extremal Decorrelated scenario that in such case the function $\tilde{f}$ is increasing and has its minimum (which is not necessarily a critical point) in $z=0$. So in the following we will always consider $b<\tilde{G}(\gamma)$.

Remember the definition of $\eta$ and $j$ in eq. 15 and the fact that we use' to indicate the derivative with respect to $z$.

The proof of the uniqueness of the critical point of $\tilde{\phi}$ still holds for $b=0$ and $b=\tilde{G}(\gamma)$, and we proved $z^{*}(\gamma, \tilde{G}(\gamma))=z_{+}^{*}(G(\gamma))$. In particular, the single point of minimum $z^{*}(\gamma, b)$ defined above of $\tilde{\phi}$ lies in $] 0, z_{2}[$. 
In a following paragraph we show that the only solution to $p(z)=q(z)=0$ is for $\hat{\gamma}=-\sqrt{\frac{9+5 \sqrt{3}}{18}}$ at $\hat{z}=\sqrt{\frac{3-\sqrt{3}}{2}}$. Take $\gamma \neq \hat{\gamma}$. With reference to the observations in section 2.3. we can conclude that the set $Z(\gamma)$ of the critical points of $\tilde{\phi}$ is equal to the interval with extrema the only zero of $p=\eta j_{2}^{\prime}-2 \eta^{\prime} j_{2}$, denoted $z^{*}(\gamma, 0)$, and the point $z^{*}(\gamma, \tilde{G}(\gamma))$.

For $\gamma=\hat{\gamma}$, the minimum point of $\tilde{\phi}$ does not depend on $b$ and it is $z^{*}(\hat{\gamma}, b)=\hat{z}$. In such case, $\tilde{G}(\hat{\gamma})=2 \sqrt{3 \sqrt{3}-5}$. In the following we see what happens for the other values of $\gamma$.

It holds $q(z)=-\frac{2 z^{4} \gamma^{2}\left(z^{2}-3\right)+4 z^{3} \gamma\left(z^{2}-3\right)+3 z^{4}-8 z^{2}+1}{8 \sqrt{1-z^{2}}(\gamma z+1)^{2}}$. Suppose $z_{q}(\gamma)$ is a zero of $q$, then solving the equation $q\left(z_{q}\right)=0$ in $\gamma$, the only solution greater than -1 is $\gamma=\gamma_{q}\left(z_{q}\right):=\frac{1-z_{q}^{2}}{z_{q}^{2} \sqrt{2\left(3-z_{q}^{2}\right)}}-\frac{1}{z_{q}}$. It can be shown that $\gamma_{q}^{\prime}(z)=\frac{1}{z^{2}}\left(1-\frac{z^{4}-3 z^{2}+6}{z \sqrt{2}\left(3-z^{2}\right)^{\frac{3}{2}}}\right)<0$ for every $\left.z \in\right] 0,1\left[\right.$. This means that $\gamma_{q}$ is invertible from $] 0,1[$ to $]-1, \infty\left[\right.$ and that for fixed $\gamma, q$ has a unique zero. Observe that $\gamma_{q}(\hat{z})=\hat{\gamma}$. Since $q(0)<0$, on the left of its zero $z_{q}(\gamma), q$ is negative while on the right it is positive. Also, if $\gamma<\hat{\gamma}$, then $z_{q}(\gamma)>\hat{z}$. We now look at $p(z)=\frac{2 \gamma^{2} z^{6}+12 \gamma^{3} z^{5}+3 z^{4}\left(10 \gamma^{2}-1\right)+28 \gamma z^{3}+12 z^{2}-1}{4(\gamma z+1)^{3}}$. Substituting $\gamma$ with $\gamma_{q}(z)$ in the last expression, we find $p\left(z ; \gamma=\gamma_{q}(z)\right)=-\frac{z^{2}\left(2 z^{4}-3 z^{2}-3+z \sqrt{2}\left(3-z^{2}\right)^{\frac{3}{2}}\right)}{1-z^{2}}$. We call $r(z):=2 z^{4}-3 z^{2}-3+z \sqrt{2}\left(3-z^{2}\right)^{\frac{3}{2}}$, then $r(\hat{z})=r(1)=0$ and $r(0)=-3<0$. Furthermore $r^{\prime}(z)=-\left(4 z^{2}-3\right)\left(\sqrt{2\left(3-z^{2}\right)}-2 z\right)$, where the second factor is positive for $z<1$, so $r$ is increasing in $\left[0, \frac{\sqrt{3}}{2}[\ni \hat{z}\right.$ and decreasing in $] \frac{\sqrt{3}}{2}, 1[$, with unique zeros at $\hat{z}$ and 1 . This means that for the previous choice of $\gamma<\hat{\gamma}, p\left(z_{q}(\gamma)\right)<0$, so $z^{*}(\gamma, 0)>z_{q}(\gamma)$ because $z^{*}(\gamma, 0)$ is the unique zero of $p$. Remember that $q$ is positive in $] z_{q}(\gamma), z^{*}(\gamma, 0)$. Since $b^{*}$ defined in eq. (4) lives where $p$ and $q$ have the same sign and since the set $Z(\gamma)$ is an interval, the latter will lie on the right of $z^{*}(\gamma, 0)$, indeed on the immediate left we have seen that $p$ is negative while $q$ is positive. The reasoning is similar for $\gamma>\hat{\gamma}$, with the conclusion that the previous set is on the left of $z^{*}(\gamma, 0)$.

Limits of $z^{*}$ with respect to $b$ When $b$ goes to 0 and $\gamma$ is not $\hat{\gamma}$, the value of $z^{*}(\gamma, b)$ is the only solution smaller than $z_{2}(\gamma)$ to $p(z)=0$, or

$$
2 \gamma^{2} z^{6}+12 \gamma^{3} z^{5}+3 z^{4}\left(10 \gamma^{2}-1\right)+28 \gamma z^{3}+12 z^{2}-1=0
$$

Since the equation is of sixth degree, the solution cannot be written explicitly, but it can be found by a numeric routine in the interval $] 0, z_{2}(\gamma)[$.

Another cunning way to find $z^{*}(\gamma, 0)$ is to use again the trick of changing the role between parameters and variables. Firstly, note that if $\gamma=0$, the only solution to eq. 20 is $z=\frac{1}{\sqrt{6+\sqrt{33}}}$. If $\gamma \neq 0$, substitute $z=\frac{u}{\gamma}$ in the above equation, then we look for $u$ solving

$$
\gamma^{4}-2 u^{2}\left(6 u^{3}+15 u^{2}+14 u+6\right) \gamma^{2}+u^{4}\left(3-2 u^{2}\right)=0
$$

The interval where $u$ lives is such that $0<\frac{u}{\gamma}<z_{2}(\gamma)$, or equivalently $\frac{\gamma z_{2}(\gamma)}{u}>1$. If $u$ is negative, it must hold $0>u>\gamma z_{2}(\gamma)>\gamma>-1$, so for sure $u>-1$. Imagine to fix $u$, then equation eq. 21 is quadratic in $\gamma^{2}$ and has four (eventually complex) solutions $\pm \sqrt{\Gamma_{ \pm}}$, with $\Gamma_{ \pm}=u^{2}\left(6 u^{3}+\right.$ $\left.15 u^{2}+14 u+6 \pm(1+u)^{2} \sqrt{3\left(12 u^{2}+12 u+11\right)}\right)$. The solution $\gamma$ must have the same sign of $u$, since $z>0$. If $u$ is positive, the solution $\sqrt{\Gamma_{-}}$is smaller than $u$, so also $\sqrt{\Gamma_{-}} z_{2}\left(\sqrt{\Gamma_{-}}\right)$is smaller than $u$. Then, the only possible solution could be $\sqrt{\Gamma_{+}}$. We plot in Figure 7 (left) the graph of 

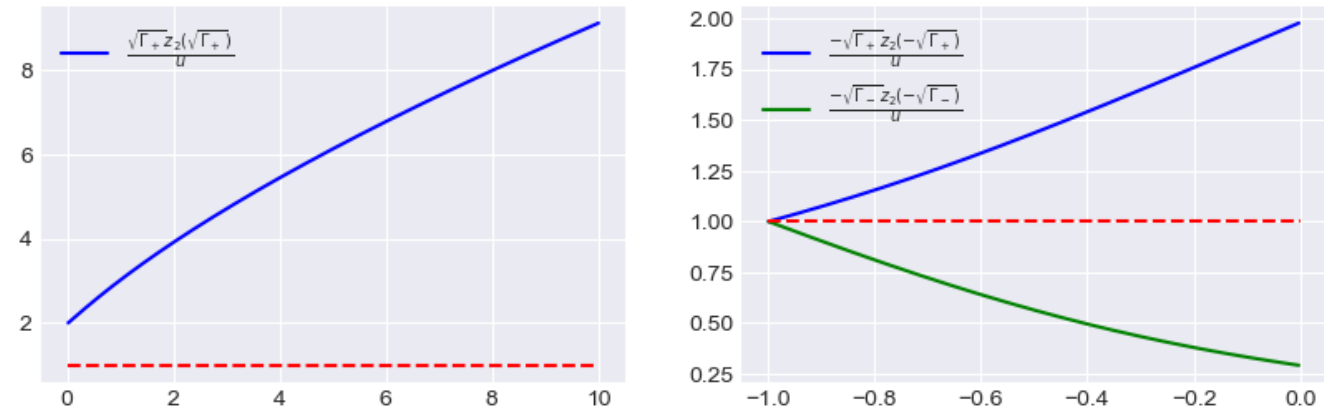

Figure 7: Study of $\frac{ \pm \sqrt{\Gamma_{ \pm}} z_{2}\left( \pm \sqrt{\Gamma_{ \pm}}\right)}{u}$ as functions of $u$.

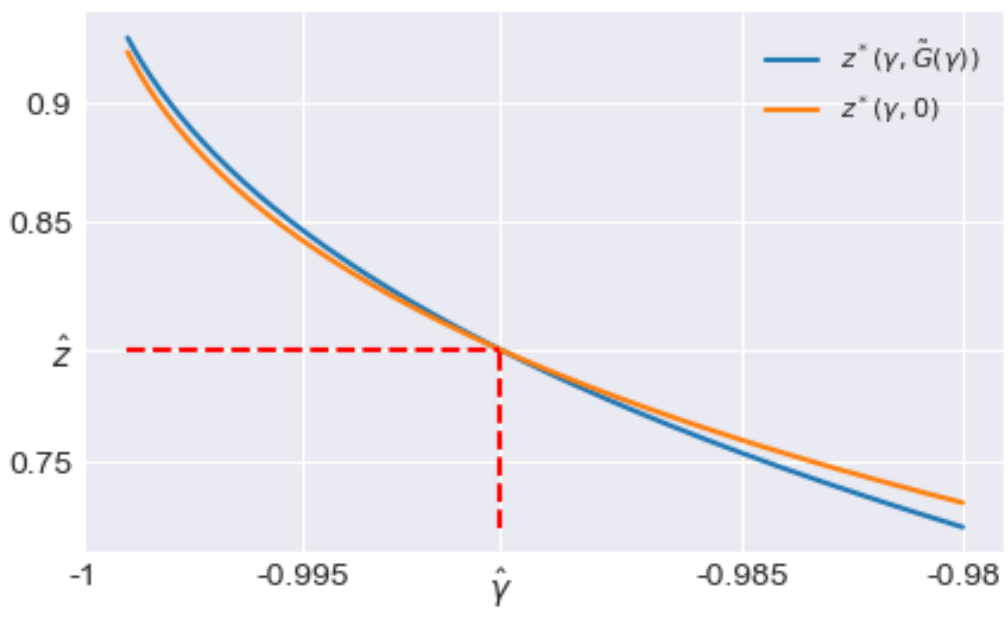

Figure 8: $z^{*}(\gamma, 0)$ and $z^{*}(\gamma, \tilde{G}(\gamma))$ as functions of $\gamma$ ranging from -1 to -0.98 .

$\frac{\sqrt{\Gamma_{+}} z_{2}\left(\sqrt{\Gamma_{+}}\right)}{u}$ to check whether it is greater than 1. If $u$ is negative, we plot in Figure 7 (right) the graphs of $\frac{-\sqrt{\Gamma_{ \pm}} z_{2}\left(-\sqrt{\Gamma_{ \pm}}\right)}{u}$ and check again whether these quantities are greater than 1 .

We can see that for every $u>0$, the solution $\sqrt{\Gamma_{+}}$is admissible and that for $-1<u<0$, the only admissible solution is $-\sqrt{\Gamma_{+}}$. To sum up, for $u>-1$, the solution to eq. 21 in terms of $\gamma$ is eq. 16 and it ranges from -1 to $\infty$. Observe that if $u=0$, the value of $\frac{u}{\gamma^{*}(u)}$ is exactly $\frac{1}{\sqrt{6+\sqrt{33}}}$, so the definition is well posed even in this case.

\subsubsection{Numerical illustration of the interval for $z^{*}$}

We report in Figures 8 and 9 the graphs of $z^{*}(\gamma, 0)$, which is the only zero of $p(z, \gamma)$ in $] 0, z_{2}(\gamma)[$, and of $z^{*}(\gamma, \tilde{G}(\gamma))$. 


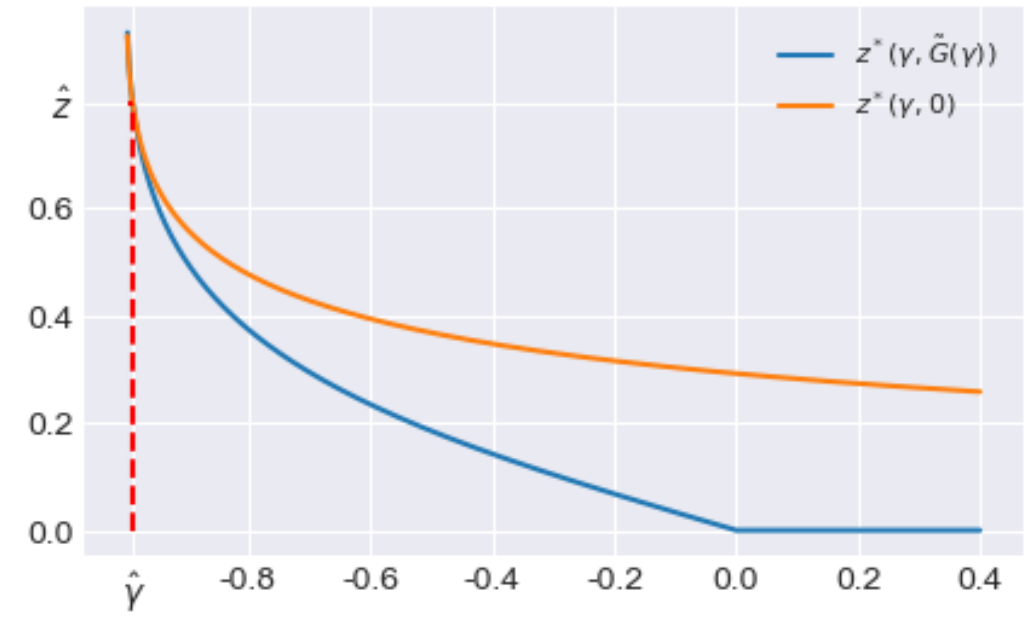

Figure 9: $z^{*}(\gamma, 0)$ and $z^{*}(\gamma, \tilde{G}(\gamma))$ as functions of $\gamma$ ranging from -1 to 0.4 .

\section{SSVI}

The SSVI is a sub-SVI classically parametrized as

$$
S S V I(k ; \theta, \varphi, \rho)=\frac{\theta}{2}\left(1+\rho \varphi k+\sqrt{(\varphi k+\rho)^{2}+1-\rho^{2}}\right) .
$$

It can be easily checked that the corresponding SVI parameters are

$(a, b, m, \rho, \sigma)=\left(\frac{\theta\left(1-\rho^{2}\right)}{2}, \frac{\theta \varphi}{2},-\frac{\rho}{\varphi}, \rho, \frac{\sqrt{1-\rho^{2}}}{\varphi}\right)$, so that $\gamma=\sqrt{1-\rho^{2}}$ and the special property $\mu=$ $-\frac{\rho}{\sqrt{1-\rho^{2}}}=l^{*}$ hold, where $l^{*}$ is the unique critical point of the smile, which is a point of minimum, of $N$. The corresponding SVI formula becomes

$$
S V I\left(k ; b \sigma \sqrt{1-\rho^{2}}, b, \rho,-\frac{\rho \sigma}{\sqrt{1-\rho^{2}}}, \sigma\right)=b \sigma\left(\sqrt{1-\rho^{2}}+\rho\left(\frac{k}{\sigma}+\frac{\rho}{\sqrt{1-\rho^{2}}}\right)+\sqrt{\left(\frac{k}{\sigma}+\frac{\rho}{\sqrt{1-\rho^{2}}}\right)^{2}+1}\right) .
$$

With our notations, $N(l)=\sqrt{1-\rho^{2}}+\rho l+\sqrt{l^{2}+1}$.

We plot in Figure 10 an SSVI with $b=1, \rho=\frac{1}{2}$ and $\sigma=\frac{1}{2}$.

\subsection{The Fukasawa conditions}

Since $\gamma>0$, we always have $\gamma>\tilde{F}(b, \rho)$. We show that also the condition on $\mu$ is verified for $\gamma>0$.

Lemma 6.1. Assume $\gamma>0$. Then for every $S V I, l^{*} \in I_{\gamma, b, \rho}$.

Proof. Consider the case $\rho \geq 0$, so that $l^{*} \leq 0$. Note that $N(l)-\gamma=l N^{\prime}(l)+N^{\prime \prime}(l)\left(l^{2}+1\right)$. Then the condition $L_{-}(l)<l^{*}$ for $l<l^{*}$ becomes $\frac{\gamma+l N^{\prime}(l)+N^{\prime \prime}(l)\left(l^{2}+1\right)}{2 N^{\prime}(l)}\left(4+b N^{\prime}(l)\right)<l+l^{*}$ or equivalently $\gamma>\frac{N^{\prime}(l)}{4+b N^{\prime}(l)}\left(2 l^{*}-l\left(2+b N^{\prime}(l)\right)\right)-N^{\prime \prime}(l)\left(l^{2}+1\right)$. 


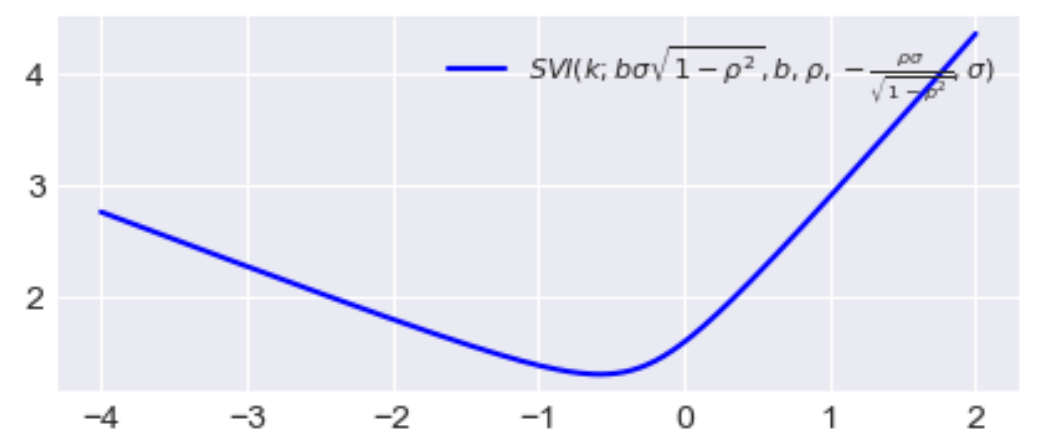

Figure 10: SSVI with $b=1, \rho=\frac{1}{2}$ and $\sigma=\frac{1}{2}$.

The term $-N^{\prime \prime}(l)\left(l^{2}+1\right)$ is strictly negative and if $2 l^{*}-l\left(2+b N^{\prime}(l)\right) \geq 0$, also the first term is negative, so that if $\gamma \geq 0$ we automatically have $L_{-}(l)<l^{*}$ for every $l<l^{*}$. When $\rho>0$ (or $\rho=0$ and $b<2)$, since $b(1+\rho) \leq 2$, it holds $b(1-\rho) \neq 2$. Because $L_{-}(-\infty)=L_{-}\left(l^{*-}\right)=-\infty$ and $L_{-}$is continuous, it holds $\sup _{l<l^{*}} L_{-}(l)=L_{-}\left(l_{-}\right)$where $\left.l_{-} \in\right]-\infty, l^{*}\left[\right.$. Then if we show $L_{-}(l)<l^{*}$ for every $l<l^{*}$, it follows $l^{*}>\sup _{l<l^{*}} L_{-}(l)$. If $\rho=0$ and $b=2$, it holds $b(1-\rho)=2$, so $l_{-}=-\infty$ and $L_{-}\left(l_{-}\right)=-\gamma$. In such case, the only annoying situation arises for $\gamma=0$, because it holds $l^{*}=0=\sup _{l<l^{*}} L_{-}(l)$. Taking $\gamma>0$ this case does not arise.

Consider the quantity $2 l^{*}-l\left(2+b N^{\prime}(l)\right)$. Since $b(1+\rho) \leq 2$, it is greater than $-2\left(-l^{*}+\right.$ $\left.l\left(1+\frac{N^{\prime}(l)}{1+\rho}\right)\right)$, which is positive iff, substituting and dividing by -2 , the quantity $c(l):=\frac{\rho}{\sqrt{1-\rho^{2}}}+$ $\frac{l}{1+\rho}\left(1+2 \rho+\frac{l}{\sqrt{l^{2}+1}}\right)$ is negative. Observe that for $l$ going to $-\infty, c$ goes to $-\infty$ when $\rho>0$ and to $0^{-}$when $\rho=0$. Furthermore $c\left(l^{*}\right)=0$. The derivative of $c$ is $c^{\prime}(l)=\frac{1}{1+\rho}\left(1+2 \rho+\frac{l\left(l^{2}+2\right)}{\left(l^{2}+1\right)^{\frac{3}{2}}}\right)$. We would like $c^{\prime}$ to be positive, so that $c$ would be increasing and, from the previous analysis, negative for $l<l^{*}$. However, this does not always happen. Indeed, the minimum point of $c^{\prime}$ is reached at $-\sqrt{2}$ and it is $\frac{1}{1+\rho}\left(1+2 \rho-\frac{4}{3} \sqrt{\frac{2}{3}}\right)$, which is non-negative iff $\rho \geq\left(\frac{2}{3}\right)^{\frac{3}{2}}-\frac{1}{2}=\bar{\rho}$. Therefore, we are annoyed by the cases where $\rho<\bar{\rho}$. Under this hypothesis, call $\bar{l}(\rho)$ the first zero of $c^{\prime}$, that is also the only zero of $c^{\prime}$ smaller than $-\sqrt{2}$. We need to prove that $d(\rho):=c(\bar{l}(\rho) ; \rho)<$ 0 . Observe that $\frac{d}{d \rho} d(\rho)=\partial_{\rho} c(\bar{l}(\rho) ; \rho)+c^{\prime}(\bar{l}(\rho) ; \rho) \frac{d}{d \rho} \bar{l}(\rho)=\partial_{\rho} c(\bar{l}(\rho) ; \rho)$. The latter quantity is $\frac{1}{\left(1-\rho^{2}\right)^{\frac{3}{2}}}+\frac{l}{(1+\rho)^{2}}\left(1-\frac{l}{\sqrt{l^{2}+1}}\right)$, which is increasing in $l$ and so it is smaller than its value at $l=-\sqrt{2}$. Then $\partial_{\rho} c(\bar{l}(\rho) ; \rho) \leq \frac{1}{\left(1-\rho^{2}\right)^{\frac{3}{2}}}-\frac{\sqrt{2}}{(1+\rho)^{2}}\left(1-\sqrt{\frac{2}{3}}\right)$ and it is negative for $\rho<\bar{\rho}$. Then, it is enough to show $d(0)<0$. For $\rho=0$, we have $\bar{l}(0)=-\infty$ and it is easy to see that $d(0)=0^{-}$as desired.

Remember that if $l^{*}>0$ (i.e. $\rho<0$ ), then $l^{*}>L_{-}\left(l_{-}\right)$because $L_{-}\left(l_{-}\right) \leq 0$ for Lemma 4.4 in 6]. Then we have proven that $\sup _{l<l^{*}} L_{-}(l)<l^{*}$ for every $\gamma>0$ and $\rho$.

Using the symmetries, we have that for every $\rho$

$$
l^{*}(\rho)>L_{-}\left(l_{-}(\rho) ; \rho\right)=-L_{+}\left(-l_{-}(\rho) ;-\rho\right)=-L_{+}\left(l_{+}(-\rho) ;-\rho\right)
$$

or equivalently $l^{*}(-\rho)<L_{+}\left(l_{+}(-\rho) ;-\rho\right)$. 
In particular this lemma holds for SSVI and has as immediate consequence the following:

Lemma 6.2 (Fukasawa conditions for SSVI). An SSVI with $0<b \leq \frac{2}{1+|\rho|}$ always satisfies the Fukasawa conditions.

\subsection{The condition on $\sigma$}

As in the previous chapters, we start this section by defining some (already used) useful functions:

$$
\begin{aligned}
b^{*}(l, \rho) & :=\sqrt{\frac{h(l, \rho)\left(h(l, \rho) g_{2}^{\prime}(l, \rho)-2 h^{\prime}(l, \rho) g_{2}(l, \rho)\right)}{g(l, \rho)\left(g(l, \rho) g_{2}^{\prime}(l, \rho)-2 g^{\prime}(l, \rho) g_{2}(l, \rho)\right)}}, \\
\sigma^{*}(l, \rho) & :=-\frac{b^{*}(l, \rho) g_{2}(l, \rho)}{2\left(h(l, \rho)^{2}-b^{*}(l, \rho)^{2} g(l, \rho)^{2}\right)}
\end{aligned}
$$

We will show that the only positive zero of $g_{2}$ is

$$
l_{2}(\rho)=\frac{1}{\tan \left(\frac{\arccos (-\rho)}{3}\right)} .
$$

The objective of this subsection is to prove the following:

Proposition 6.3 (Fully explicit no arbitrage domain for SSVI). If an SSVI is arbitrage-free then $0<b \leq \frac{2}{1+|\rho|}$.

- If $b=\frac{2}{1+|\rho|}$, an SSVI is arbitrage-free iff $\sigma \geq \sqrt{1-\rho^{2}}$.

- If $b<\frac{2}{1+|\rho|}$, for $\rho \geq 0$, define $\bar{l}(0, \rho)$ the only root in $] l_{2}(\rho), \infty\left[\right.$ of $b^{*}(l, \rho)=0$.

Then, under the assumption that $\tilde{f}$ has a unique critical point (that we sustain numerically in Appendix A):

$\square$ an SSVI with $\rho \geq 0$ is arbitrage-free iff it can be parametrized as

$$
S V I(k)=\sigma b^{*}(l, \rho)\left(\sqrt{1-\rho^{2}}+\rho\left(\frac{k}{\sigma}+\frac{\rho}{\sqrt{1-\rho^{2}}}\right)+\sqrt{\left(\frac{k}{\sigma}+\frac{\rho}{\sqrt{1-\rho^{2}}}\right)^{2}+1}\right)
$$

where $l \in\left[\bar{l}(0, \rho), \infty\left[\right.\right.$ and $\sigma \geq \sigma^{*}(l, \rho)$;

$\square$ an SSVI with $\rho<0$ is arbitrage-free iff it can be parametrized as

$$
S V I(k)=\sigma b^{*}(l,-\rho)\left(\sqrt{1-\rho^{2}}+\rho\left(\frac{k}{\sigma}+\frac{\rho}{\sqrt{1-\rho^{2}}}\right)+\sqrt{\left(\frac{k}{\sigma}+\frac{\rho}{\sqrt{1-\rho^{2}}}\right)^{2}+1}\right)
$$

where $l \in\left[\bar{l}(0,-\rho), \infty\left[\right.\right.$ and $\sigma \geq \sigma^{*}(l,-\rho)$. 


\subsubsection{Proof of Proposition 6.3}

We give the proof in the case $\rho \geq 0$. Using Lemmas 2.4 and 2.5 we get the following remark:

Remark 6.4. Suppose $\rho \geq 0$, then $l_{2} \leq-l_{1}$. Fix $l>l_{2}>0$. For the particular case of SSVI, $h(-l)-h(l)=0$ so the difference between $G_{1}(l)$ and $G_{1}(-l)$ is given by $-b^{2} \frac{\rho l}{4 \sqrt{l^{2}+1}}$, which is negative. Since $g_{2}(l) \leq g_{2}(-l)$, then $\tilde{f}(l) \leq \tilde{f}(-l)$, so $\inf _{l>l_{2}} \tilde{f}(l) \leq \inf _{l<l_{1}} \tilde{f}(l)$. It follows then that $\sigma^{*}=\frac{b}{2 \inf _{l}>l_{2} \tilde{f}(l)}$.

Similarly for $\rho<0$ and $l<l_{1}$, it holds $\tilde{f}(l)<\tilde{f}(-l)$ and $\sigma^{*}=\frac{b}{2 \inf _{l<l_{1}} \tilde{f}(l)}$.

Study of $j_{2}$ In this section, we work with $x=\frac{l}{\sqrt{l^{2}+1}}$, whence

$$
\begin{aligned}
\sqrt{1-x^{2}} N(l(x)) & =1+\rho x+\sqrt{1-\rho^{2}} \sqrt{1-x^{2}}, \\
N^{\prime}(l(x)) & =x+\rho, \\
N^{\prime \prime}(l(x)) & =\left(1-x^{2}\right)^{\frac{3}{2}} .
\end{aligned}
$$

Remark 6.5. We redefine the useful functions when using the $x$ variable and set:

$$
\Pi(x):=N(l(x)), j_{2}(x):=g_{2}(l(x)), j(x):=g(l(x)), J_{1}(x):=G_{1}(l(x)), \tilde{\phi}(x):=\tilde{f}(l(x)) .
$$

The derivative with respect to $l$ is indicated with', so for example the function $j_{2}^{\prime}(x)$ corresponds to $g_{2}^{\prime}(l(x))$ rather than $\frac{d j_{2}}{d x}(x)$.

In the study of the function $g_{2}$ in $\left[\right.$, we recall that the function has one positive zero $l_{2}>l^{*}$ and it has at least one point of minimum (that here we prove to be unique and call $m_{2}$ ) at the right of $l_{2}$.

Computation of $l_{2} \quad$ Let us study the location of the unique positive zero of $j_{2}(x)=(1-$ $\left.x^{2}\right)^{\frac{3}{2}}-\frac{(x+\rho)^{2} \sqrt{1-x^{2}}}{2\left(1+\rho x+\bar{\rho} \sqrt{1-x^{2}}\right)}$, where $\bar{\rho}=\sqrt{1-\rho^{2}}$. Note that $j_{2}(1)=0$; so for $x<1, j_{2}(x)=0$ iff

$$
2\left(1-x^{2}\right)\left(1+\rho x+\bar{\rho} \sqrt{1-x^{2}}\right)=(x+\rho)^{2} .
$$

Isolating the radical and squaring yields that the zeros of $j_{2}$ solve the polynomial root equation

$$
4 \bar{\rho}^{2}\left(1-x^{2}\right)^{3}=\left((x+\rho)^{2}-2(1+\rho x)\left(1-x^{2}\right)\right)^{2} .
$$

The key observation is that $-\rho$ is a root of eq. $(29)$ and not of eq. $(28)$. This leads to the following factorization of the polynomial $(x+\rho)\left(4 x^{5}+8 \rho x^{4}+\left(4 \rho^{2}-3\right) x^{3}-5 \rho x^{2}-\rho^{2} x+\rho^{3}\right)$. Now $-\rho$ is again a root of the rightmost factor which factors in turn in $(x+\rho)\left(4 x^{4}+4 \rho x^{3}-3 x^{2}-2 \rho x+\rho^{2}\right) \ldots$ and the miracle continues! Indeed, $-\rho$ is again a root of this later factor, leading to the fact that the zeros of $j_{2}$ solve $\rho=x\left(3-4 x^{2}\right)$.

In particular:

- $\rho=0$, assuming we can exclude $x=0$, gives $x=\frac{\sqrt{3}}{2}<1$

- $\rho=1$ gives the polynomial equation $4 x^{3}-3 x+1=0$ which reads $4(x+1)\left(x-\frac{1}{2}\right)^{2}=0$, whence $x=\frac{1}{2}$. 
Taking the derivative with respect to $\rho$ gives $1=3 x^{\prime}(\rho)\left(1-4 x(\rho)^{2}\right)$ which gives in turn that $\rho \rightarrow x(\rho)$ is decreasing when $x>\frac{1}{2}$. We can now either solve the 3rd degree polynomial root problem, or take $x$ as a parameter varying in the range $\left[\frac{1}{2}, \frac{\sqrt{3}}{2}\right]$, and backup $\rho$ by the formula $\rho(x)=3 x-4 x^{3}$.

Going the other route, we note that the polynomial $Q_{\rho}(x):=4 x^{3}-3 x+\rho$ satisfies $Q_{\rho}\left(\frac{1}{2}\right)=$ $-1+\rho<0, Q_{\rho}\left(\frac{\sqrt{3}}{2}\right)=\rho \geq 0$ and also $Q_{\rho}^{\prime}(x)=3\left(4 x^{2}-1\right)$ which is positive in the range $\left.] \frac{1}{2}, \frac{\sqrt{3}}{2}\right]$, so it has a unique real root in this range. Its unique local maximum is located at $x=-\frac{1}{2}$ where $Q_{\rho}\left(-\frac{1}{2}\right)=1+\rho>0$, with, together with the observation that $Q_{\rho}(-1)=\rho-1<0$ and $Q_{\rho}\left(\frac{1}{2}\right)<0$, gives that $Q_{\rho}$ has 2 other real roots located in $]-1,-\frac{1}{2}[$ and $]-\frac{1}{2}, \frac{1}{2}[$, so that $x(\rho)$ is the largest of the roots of $Q_{\rho}$. The three solutions have explicit formula

$$
\cos \left(\frac{\arccos (-\rho)-2 \pi k}{3}\right), \quad k=0,1,2
$$

and the greatest is $x(\rho)=\cos \left(\frac{\arccos (-\rho)}{3}\right)$. It follows that $l_{2}(\rho)$ is given by eq. 25.

Uniqueness of $m_{2}$ Using the parametrization in $x$, we get that the zeros of $j_{2}^{\prime}$ solve:

$$
(x+\rho)^{3}=2\left(1-x^{2}\right)\left(\rho x+\bar{\rho} \sqrt{1-x^{2}}+1\right)\left(3 x\left(\rho x+\bar{\rho} \sqrt{1-x^{2}}+1\right)+x+\rho\right) .
$$

In particular for $\rho=0$ this reads $x^{2}=2\left(1-x^{2}\right)\left(1+\sqrt{1-x^{2}}\right)\left(4+3 \sqrt{1-x^{2}}\right)$. Isolating the radical and squaring yields the polynomial equation $\left(x^{2}+2\left(1-x^{2}\right)\left(3 x^{2}-7\right)\right)^{2}=4 \times 49\left(1-x^{2}\right)^{3} ; 0$ is a root, whereas it is not a solution of eq. 30 . Letting $X=1-x^{2}$, the polynomial factorizes into $(1-X)^{2}\left(36 X^{2}-16 X+1\right)$, yielding two roots in $] 0,1\left[, \frac{4+\sqrt{7}}{18}\right.$ and $\frac{4-\sqrt{7}}{18}$, with only the latter one solving eq. 30 , giving in turn $x_{m_{2}}(\rho=0)=\sqrt{\frac{\sqrt{7}}{18}+\frac{7}{9}}$.

Let us turn now to $\rho=1$. In this case eq. (30) simplifies to $1=2(1-x)(3 x+1)$, or yet $6 x^{2}-4 x-1=0$, yielding $x_{m_{2}}(\rho=1)=\frac{2+\sqrt{10}}{6}$.

Before investigating the general case, we can observe that if we set $x=\rho$ in eq. (30) we get an equation in $\rho$ which is $8 \rho^{3}=4\left(1-\rho^{2}\right) \times 8 \rho$, or yet $\rho^{2}=4\left(1-\rho^{2}\right)$ which gives $x_{m_{2}}\left(\rho=\frac{2}{\sqrt{5}}\right)=\frac{2}{\sqrt{5}}$.

Let us prove now that in the general case $g_{2}$ has a unique critical point.

Note that $\partial_{\rho} N(l)=l+l^{*}$, so $\partial_{\rho} g_{2}(l)=-\partial_{\rho} \frac{N^{\prime}(l)^{2}}{2 N(l)}=-\frac{N^{\prime}(l)}{N(l)}\left(1-\frac{N^{\prime}(l)}{2 N(l)}\left(l+l^{*}\right)\right)=-\frac{N^{\prime}(l)}{N(l)} h(l)<0$ because $h(l)=\left(G_{1+}(l)+G_{1-}(l)\right) / 2>0$. From the formula $g_{2}^{\prime}(l)=N^{\prime \prime \prime}(l)-\frac{N^{\prime}(l)}{N(l)} g_{2}(l)$, we find $\partial_{\rho} g_{2}^{\prime}(l)=-\left(\partial_{\rho} \frac{N^{\prime}(l)}{N(l)}\right) g_{2}-\frac{N^{\prime}(l)}{N(l)} \partial_{\rho} g_{2}$ since $N^{\prime \prime \prime}$ does not depend on $\rho$. The second term is positive while the first has the factor $\partial_{\rho} \frac{N^{\prime}(l)}{N(l)}=\frac{1}{N(l)}\left(1-\frac{N^{\prime}(l)}{N(l)}\left(l+l^{*}\right)\right)=\frac{1}{N(l) \sqrt{l^{2}+1} \sqrt{1-\rho^{2}}}>0$. Then $\partial_{\rho} g_{2}^{\prime}(l)>0$.

We have seen that for $\rho=0$ and $\rho=1$, the function $g_{2}$ has a unique critical point $m_{2}$. Since $\partial_{\rho} g_{2}^{\prime}(l)>0$, then for every $\rho<1$, the critical points of $g_{2}$ must be greater than $m_{2}(\rho=1)=\frac{2 \sqrt{2}+\sqrt{5}}{3}$. A critical point in the $l$-variable is still a critical point in the $x$-variable and viceversa, because $\frac{d f}{d x}(x)=f^{\prime}(l(x)) \frac{d l}{d x}(x)=\frac{f^{\prime}(l(x))}{\left(1-x^{2}\right)^{\frac{3}{2}}}$ with the convention ' $=\frac{d}{d l}$. Then, showing the convexity of $g_{2}$ in the $x$-variable for $x>x_{m_{2}}(\rho=1)$ automatically proves the uniqueness of its critical point in any variable (even if $g_{2}$ is not convex in the $l$-variable). 
We have $\frac{d^{2} j_{2}}{d x^{2}}(x)=\left(\frac{d l}{d x}(x)\right)^{2} j_{2}^{\prime \prime}(x)+j_{2}^{\prime}(x) \frac{d^{2} l}{d x^{2}}(x)=\left(j_{2}^{\prime \prime}(x)+3 x \sqrt{1-x^{2}} j_{2}^{\prime}(x)\right)\left(1-x^{2}\right)^{-3}$ and

$$
\begin{aligned}
j_{2}^{\prime \prime}(x)+3 x \sqrt{1-x^{2}} j_{2}^{\prime}(x)= & \left(\Pi^{i v}(x)-\frac{\Pi^{\prime \prime}(x)^{2}}{\Pi(x)}-\frac{\Pi^{\prime}(x) \Pi^{\prime \prime \prime}(x)}{\Pi(x)}+\frac{5 \Pi^{\prime}(x)^{2} \Pi^{\prime \prime}(x)}{2 \Pi(x)^{2}}-\frac{\Pi^{\prime}(x)^{4}}{\Pi(x)^{3}}\right)+ \\
& +3 x \sqrt{1-x^{2}}\left(\Pi^{\prime \prime \prime}(x)-\frac{\Pi^{\prime}(x) \Pi^{\prime \prime}(x)}{\Pi(x)}+\frac{\Pi^{\prime}(x)^{3}}{2 \Pi(x)^{2}}\right)
\end{aligned}
$$

Since $3 x \sqrt{1-x^{2}}=-\frac{\Pi^{\prime \prime \prime}(x)}{\Pi^{\prime \prime}(x)}$, the terms $-\frac{\Pi^{\prime}(x) \Pi^{\prime \prime \prime}(x)}{\Pi(x)}$ and $-3 x \sqrt{1-x^{2}} \frac{\Pi^{\prime}(x) \Pi^{\prime \prime}(x)}{\Pi(x)}$ simplify. Also, $\Pi^{i v}(x)+3 x \sqrt{1-x^{2}} \Pi^{\prime \prime \prime}(x)=3\left(1-x^{2}\right)^{\frac{5}{2}}\left(2 x^{2}-1\right)$, which is positive since $x>\frac{2+\sqrt{10}}{6}>\frac{1}{\sqrt{2}}$. The term $-\frac{\Pi^{\prime \prime}(x)^{2}}{\Pi(x)}$ becomes positive with the sum of $\frac{\Pi^{\prime}(x)^{2} \Pi^{\prime \prime}(x)}{2 \Pi(x)^{2}}$, indeed it becomes $-\frac{\Pi^{\prime \prime}(x)}{\Pi(x)}\left(\Pi^{\prime \prime}(x)-\frac{\Pi^{\prime}(x)^{2}}{2 \Pi(x)}\right)=$ $-\frac{\Pi^{\prime \prime}(x)}{\Pi(x)} j_{2}(x)>0$. Note that the remaining $4 \frac{\Pi^{\prime}(x)^{2} \Pi^{\prime \prime}(x)}{2 \Pi(x)^{2}}$ is positive. Finally, $-\frac{\Pi^{\prime}(x)^{4}}{\Pi(x)^{3}}$ compensates with $3 x \sqrt{1-x^{2}} \frac{\Pi^{\prime}(x)^{3}}{2 \Pi(x)^{2}}$ summing to

$$
\frac{\Pi^{\prime}(x)^{3}}{\Pi(x)^{2}}\left(\frac{3}{2} x \sqrt{1-x^{2}}-\frac{\Pi^{\prime}(x)}{\Pi(x)}\right)=\frac{\Pi^{\prime}(x)^{3}}{2 \Pi(x)^{3}}\left(x+3 \rho x^{2}-2 \rho+3 x \sqrt{1-\rho^{2}} \sqrt{1-x^{2}}\right)
$$

Now $3 \rho x^{2}-2 \rho$ is positive iff $x>\sqrt{\frac{2}{3}}$ and this is true, so also the previous quantity is positive. Then, since the negative terms of $\frac{d^{2} j_{2}}{d x^{2}}$ are smaller in magnitude than its positive terms, $j_{2}$ is convex in the $x$-variable.

As a consequence, $j_{2}$ has a unique critical point for $x>x_{2}$ and it lies between $x_{m_{2}}(\rho=1)$ and $x_{m_{2}}(\rho=0)$.

In order to study $x_{m_{2}}$ in the general case, let us isolate the radical of eq. (30) and square. We get the daunting polynomial root equation

$$
\left((\rho+x)^{3}-2\left(1-x^{2}\right)\left(3 \bar{\rho}^{2} x\left(1-x^{2}\right)+(\rho x+1)(3 x(\rho x+1)+(\rho+x))\right)\right)^{2}=4 \bar{\rho}^{2}\left(1-x^{2}\right)^{3}(6 x(\rho x+1)+\rho+x)^{2}
$$

There again, we observe that $-\rho$ is a root, whereas it does not solve the initial equation. This leads to an iterative factorization where eventually $-\rho$ is a root of order 4 . The remaining factor is

$$
\rho^{2}+2 \rho\left(12 x^{5}-16 x^{3}+5 x\right)+\left(36 x^{6}-56 x^{4}+21 x^{2}\right) .
$$

For $x \in\left[x_{m_{2}}(\rho=1), x_{m_{2}}(\rho=0)\right]$, the only positive $\rho$ solution is

$$
\rho(x)=x\left(-12 x^{4}+16 x^{2}-5+2\left(1-x^{2}\right) \sqrt{36 x^{4}-24 x^{2}+1}\right) .
$$

Since $\partial_{\rho} j_{2}^{\prime}<0$, also $\partial_{\rho} \frac{d j_{2}}{d x}<0$ and the function $\rho(x)$ is invertible in $\left[x_{m_{2}}(\rho=1), x_{m_{2}}(\rho=0)\right]=$ $\left[\frac{2+\sqrt{10}}{6}, \sqrt{\frac{\sqrt{7}}{18}+\frac{7}{9}}\right]$ and its inverse gives the value of $x_{m_{2}}$ for fixed $\rho$. 
Study of $J_{1} \quad$ From the above remark, $J_{1}(x)=\eta^{2}(x)-b^{2} j^{2}(x)$ where $\eta(x)=\frac{1}{2}\left(1+\sqrt{\frac{1-x^{2}}{1-\rho^{2}}}\right)$ and $j(x)=\frac{x+\rho}{4}$. The simplified formula for $\eta$ can be recovered from:

$$
\begin{aligned}
\eta(x) & =1-\frac{\Pi^{\prime}(x)}{2 \Pi(x)}\left(\frac{x}{\sqrt{1-x^{2}}}-\frac{\rho}{\sqrt{1-\rho^{2}}}\right) \\
& =1-\frac{(x+\rho)\left(x \sqrt{1-\rho^{2}}-\rho \sqrt{1-x^{2}}\right)}{2 \sqrt{1-\rho^{2}}\left(1+\rho x+\sqrt{1-\rho^{2}} \sqrt{1-x^{2}}\right)} \\
& =\frac{\sqrt{1-x^{2}}\left(\left(1-\rho^{2}\right)+\rho x+1\right)+\sqrt{1-\rho^{2}}\left(\left(1-x^{2}\right)+\rho x+1\right)}{2 \sqrt{1-\rho^{2}}\left(1+\rho x+\sqrt{1-\rho^{2}} \sqrt{1-x^{2}}\right)}
\end{aligned}
$$

and collecting at the numerator the quantities $\sqrt{1-\rho^{2}} \sqrt{1-x^{2}}$ and $\rho x+1$, one recovers the product $\left(\sqrt{1-\rho^{2}}+\sqrt{1-x^{2}}\right)\left(1+\rho x+\sqrt{1-\rho^{2}} \sqrt{1-x^{2}}\right)$ where the second factor simplifies with the denominator.

The function $j$ is of course positive and increasing in $x$, while $\eta$ is positive decreasing in $x$, since $\frac{d \eta}{d x}(x)=-\frac{x}{2 \sqrt{1-\rho^{2}} \sqrt{1-x^{2}}}<0$. Then $J_{1}$ is a decreasing function and it attains its minimum at 1 , equal to $\frac{1}{16}(2-b(1+\rho))(2+b(1+\rho))$.

Looking at the condition $\sigma \geq \sup _{x>x_{2}}-\frac{b j_{2}(x)}{2 J_{1}(x)}$, we then have that for every $x>x_{2}$, it holds $-\frac{b j_{2}(x)}{2 J_{1}(x)}<-\frac{b j_{2}\left(m_{2}\right)}{2 J_{1}(1)}=-\frac{8 b j_{2}\left(m_{2}\right)}{\left(4-b^{2}(1+\rho)^{2}\right)}$, from which one immediately finds a no arbitrage sub-domain for SSVI:

Lemma 6.6 (No arbitrage sub-domain for SSVI). Let $j_{2}$ be given as in Remark 6.5. An SSVI with $0<b(1+|\rho|)<2$ and $\sigma \geq-\frac{8 b j_{2}\left(m_{2}(|\rho|),|\rho|\right)}{\left(4-b^{2}(1+|\rho|)^{2}\right)}$ is arbitrage-free.

Uniqueness of the critical point of $\tilde{f}$ for SSVI Remember that we consider the case $\rho \geq 0$. Then, we look at the infimum of $\tilde{f}(l)$ for $l>l_{2}$, letting $b$ be eventually 0 . For $b<\frac{2}{1+\rho}$, it holds $\tilde{f}\left(l_{2}^{+}\right)=\infty=\tilde{f}(\infty)$ and $\tilde{f}>0$, so $\tilde{f}$ must have a critical point which is a point of minimum. We want to prove that in such case $\tilde{f}$ has a unique critical point in $] l_{2}, \infty\left[\right.$. When $b=\frac{2}{1+\rho}$, it can be shown that the derivative of $\tilde{f}$ vanishes at $\infty$. We prove that even in this case this is the unique point of minimum of $\tilde{f}$.

To show that $\tilde{f}$ has a unique critical point, we can prove that $\frac{d \tilde{\phi}}{d x}$ has a unique zero.

We have seen that $J_{1}$ is decreasing as a function of $x$. A critical point must satisfied $\frac{d \tilde{\phi}}{d x}=0$ or $J_{1} \frac{d j_{2}}{d x}-\frac{d J_{1}}{d x} j_{2}=0$. Looking at the sign of the previous functions, it must hold $\frac{d j_{2}}{d x}>0$ so the critical point is on the right of $x_{m_{2}}$. At the critical point, it holds $j_{2}^{2} \frac{d^{2} \tilde{\phi}}{d x^{2}}=J_{1} \frac{d^{2} j_{2}}{d x^{2}}-\frac{d^{2} J_{1}}{d x^{2}} j_{2}:=n$, so we should show that $n$ is positive for every $x>x_{m_{2}}$.

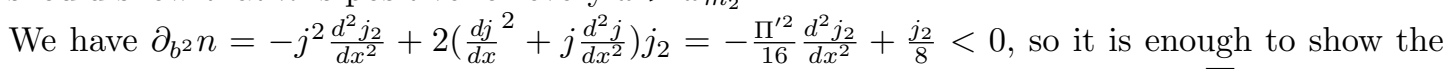
positivity of $n_{\left.\right|_{b=\frac{2}{1+\rho}}}$ for $x>x_{m_{2}}$ or, more generally, for $x>x_{m_{2}}(\rho=1)=\frac{2+\sqrt{10}}{6}$. We show it numerically in Appendix A. We plot in Figure 11 the function $n_{\left.\right|_{b=\frac{2}{1+\rho}}}$ for $x>x_{m_{2}}(\rho=1)$ for different values of $\rho$.

The $b^{*}$ approach for SSVI We have numerically shown that $\tilde{f}$ has at most one critical point in $\left.] l_{2}, \infty\right]$ and this is a point of minimum. 


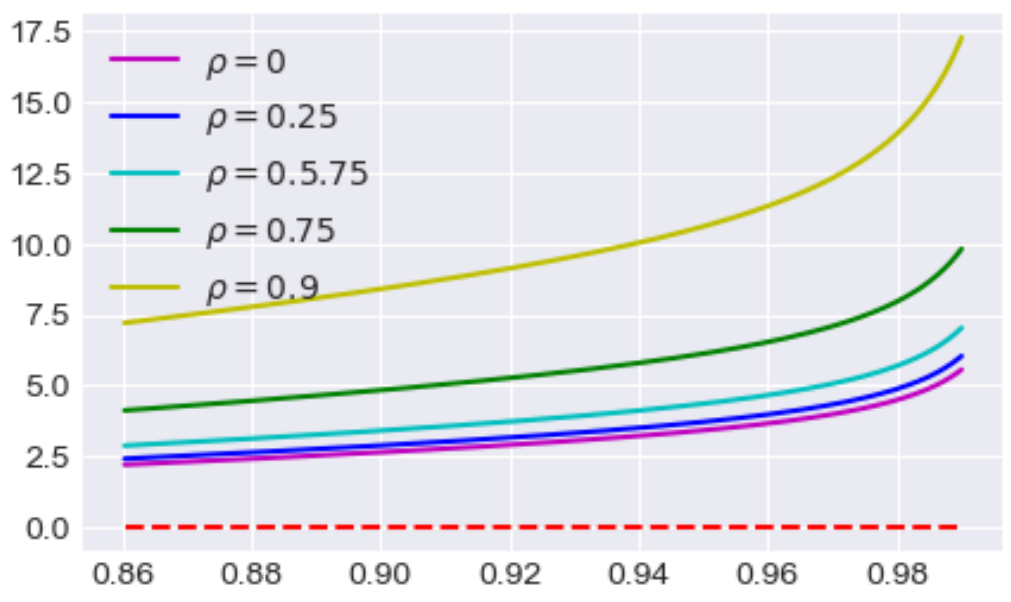

Figure 11: $\left.n^{\prime}\right|_{b=\frac{2}{1+\rho}}$ for different $\rho$ s.

- The limit of $\tilde{f}$ at $\infty$ is finite iff $b=\frac{2}{1+\rho}$ and it equals $\frac{1}{2}\left(\frac{\gamma}{1+\rho}-\mu\right)=\frac{1}{2 \sqrt{1-\rho^{2}}}$ for SSVI. So if $b=\frac{2}{1+\rho}$, the point of minimum of $\tilde{f}$ is $\infty$ because there its first derivative is null, so that due to uniqueness, the function is strictly decreasing, and in such a case, $\sigma^{*}=\sqrt{1-\rho^{2}}$.

- Consider now $b<\frac{2}{1+\rho}, \tilde{f}$ has a critical point $\left.\bar{l} \in\right] l_{2}, \infty\left[\right.$ and it satisfies $b^{2}=b^{*}(\bar{l})^{2}$ or $p(\bar{l})=$ $q(\bar{l})=0$. In the latter case, we should have $\frac{2 h^{\prime}(\bar{l})}{h(\bar{l})}=\frac{2 g^{\prime}(\bar{l})}{g(\bar{l})}$ but the right hand side is always positive while we have shown that the left hand side is negative. Then we can apply the observation in section 2.3 and obtain that the set of the critical points of $\tilde{f}$ for $\rho$ fixed is equal to the interval starting from the maximum between all the zeros of $p$, coinciding with $\bar{l}(0, \rho)$, and ending at $\bar{l}\left(\frac{2}{1+\rho}, \rho\right)=\infty$. Fix $\rho \geq 0, b<\frac{2}{1+\rho}$, choose $l \in\left[\bar{l}(0, \rho), \infty\left[\right.\right.$. Then $\sigma^{*}=\frac{b^{*}(l)}{2 \tilde{f}(l ; \rho)}$.

Limits of $\bar{l}$ Because of the uniqueness of the critical points of $\tilde{\phi}$, when $b$ goes to 0 , the critical point satisfies $\eta j_{2}^{\prime}-2 \eta^{\prime} j_{2}=0$. The numerator of $\eta j_{2}^{\prime}-2 \eta^{\prime} j_{2}$ is

$$
\begin{aligned}
& \sqrt{1-\rho^{2}}\left(-4 \rho x^{6}+2\left(6 \rho^{2}-5\right) x^{5}+24 \rho x^{4}+\left(31-14 \rho^{2}\right) x^{3}-13 \rho x^{2}+5\left(\rho^{2}-4\right) x+\rho\left(\rho^{2}-4\right)\right)+ \\
+ & \sqrt{1-x^{2}}\left(2\left(2 \rho^{2}-1\right) x^{5}+4 \rho\left(4-3 \rho^{2}\right) x^{4}+\left(21-22 \rho^{2}\right) x^{3}+\rho\left(8 \rho^{2}-15\right) x^{2}+5\left(3 \rho^{2}-4\right) x+\rho\left(3 \rho^{2}-4\right)\right) .
\end{aligned}
$$

If $\rho=0$, possible solutions are $x=0$ and $x=\sqrt{\sqrt{6}-\frac{3}{2}}$ but since $x$ must be greater than $x_{2}$, the latter is the searched solution. If $\rho=1$, the only possible solution is $x=1$. In terms of $l$, these two points correspond to $\sqrt{9+4 \sqrt{6}}$ and $\infty$.

The above expression can also be written as

$$
2 u \sqrt{1-x^{2}}\left[2 x\left(1-x^{2}\right) u-x(x+\rho)^{2}-\sqrt{1-x^{2}}(x+\rho+3 x u) v\right]+(x+\rho)^{3} v
$$


or

$$
\varphi(x):=-2 u^{2} \sqrt{1-x^{2}}\left[x \sqrt{1-x^{2}}\left(3 \sqrt{1-\rho^{2}}+\sqrt{1-x^{2}}\right)+x+\rho\right]+(x+\rho)^{3} v
$$

where $u=1+\rho x+\sqrt{1-x^{2}} \sqrt{1-\rho^{2}}$ and $v=\sqrt{1-x^{2}}+\sqrt{1-\rho^{2}}$. Observe that $(u, v)(x=1)=$ $\left(1+\rho, \sqrt{1-\rho^{2}}\right)$ and $(u, v)(x=\rho)=\left(2,2 \sqrt{1-\rho^{2}}\right)$ so that

- $\varphi(1)=(1+\rho)^{3} \sqrt{1-\rho^{2}}>0$;

- $\varphi(\rho)=-48 \rho\left(1-\rho^{2}\right)^{\frac{3}{2}} \leq 0$;

and $\varphi$ has a zero in the range $] x_{2}(\rho) \vee \rho, 1[$.

Lemma 6.7 (Computation of $\bar{l}(0, \rho)$ ). The only root of $b^{*}(l)=0$ is $\sqrt{9+4 \sqrt{6}}$ if $\rho=0, \infty$ if $\rho=1$ and, in the general case, it lies in $] l_{2}(\rho) \vee-l^{*}(\rho), \infty\left[\right.$. It can be computed as $\bar{l}(0, \rho)=\frac{\bar{x}(0, \rho)}{\sqrt{1-\bar{x}(0, \rho)^{2}}}$, where $\bar{x}(0, \rho)$ is the only zero of eq. (31), which lies in $] x_{2}(\rho) \vee \rho, 1[$.

Remember from Proposition 2.6 that the smile $S V I(a, b,-\rho,-m, \sigma)$ is arbitrage-free iff the smile $S V I(a, b, \rho, m, \sigma)$ is arbitrage-free. Furthermore, in the case of SSVI, we have the relation $S V I(k ; a, b,-\rho,-m, \sigma)=S V I(-k ; a, b, \rho, m, \sigma)$ where $a=b \sigma \sqrt{1-\rho^{2}}$ and $m=-\sigma \frac{\rho}{\sqrt{1-\rho^{2}}}$. So an SSVI with $\rho<0$ is arbitrage-free iff the SSVI with parameter $-\rho>0$ is arbitrage-free.

\subsubsection{The Gatheral-Jacquier sufficient conditions}

How does the boundary in Lemma 6.6 compare with the sufficient conditions found by Gatheral and Jacquier in Theorem 4.2 of [3]? The theorem asserts that an SSVI is free of Butterfly arbitrage if $\theta \varphi(1+|\rho|)<4$ and $\theta \varphi^{2}(1+|\rho|) \leq 4$. In terms of the SVI parameters, we have $\theta \varphi=2 b$ and $\varphi=\frac{\sqrt{1-\rho^{2}}}{\sigma}$ so that the two conditions become

$$
\begin{aligned}
& b(1+|\rho|)<2, \\
& \sigma \geq \frac{b}{2}(1+|\rho|) \sqrt{1-|\rho|^{2}},
\end{aligned}
$$

where the first is the strict Roger-Lee condition. Note that when $\sigma \geq \frac{2}{b} \sqrt{\frac{1-|\rho|}{1+|\rho|}}$ and the Roger-Lee condition is satisfied, then the second condition is automatically verified and the SSVI is free of arbitrage. Then, we can simply look at the second condition, keeping in mind that the Roger-Lee condition $b(1+|\rho|) \leq 2$ must hold, being a necessary condition.

We can compare with some plots the Gatheral-Jacquier second sufficient condition with the sufficient condition of Lemma 6.6 and the necessary and sufficient condition $\sigma \geq \sigma^{*}$. In the first couple of graphs in Figure $12, \rho$ is fixed while $b$ ranges in $] 0, \frac{2}{1+|\rho|}[$; in the second couple in Figure 13 $b$ is fixed and $\rho$ ranges in $] 0,1 \wedge\left(\frac{2}{b}-1\right)[$.

We can see that the sufficient condition of Lemma 6.6 is weaker than the Gatheral-Jacquier's one as soon as $b(1+|\rho|)$ remains low, but when this quantity goes to 2 , the former goes to $\infty$ since $J_{1}(1)$ goes to 0. 

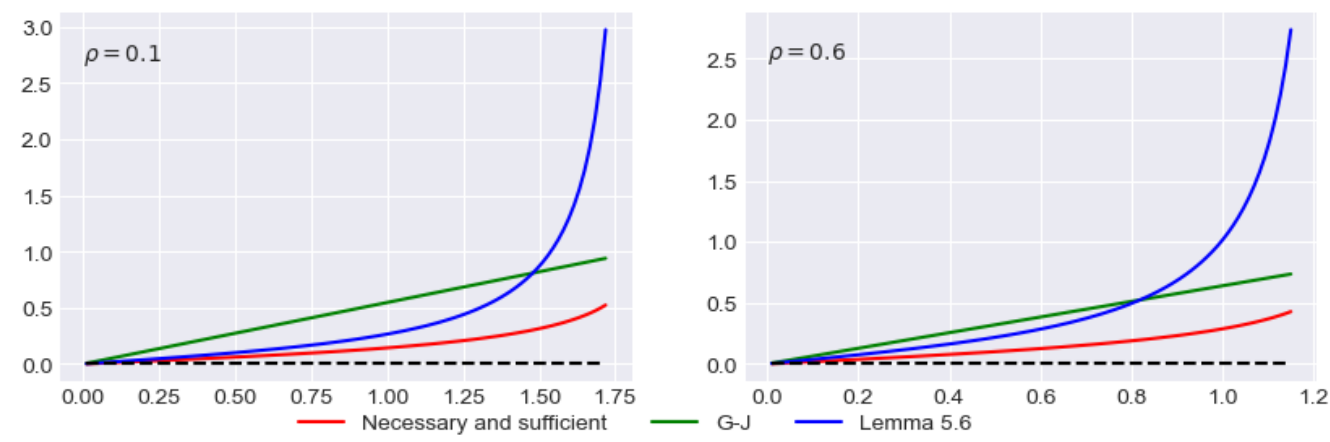

Figure 12: Comparison of Gatheral-Jacquier and Lemma 6.6 sufficient conditions as functions of $b$.
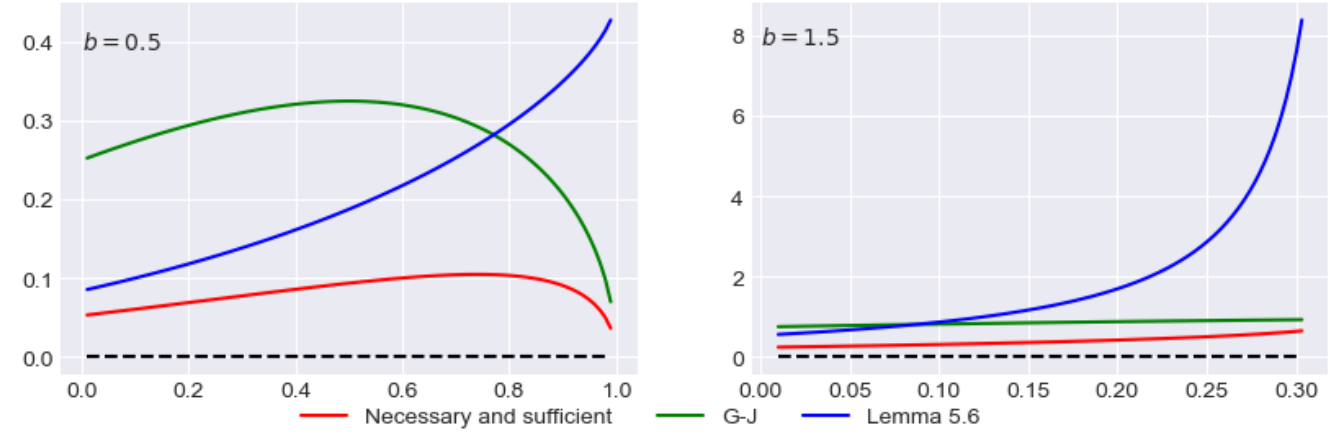

Figure 13: Comparison of Gatheral-Jacquier and Lemma 6.6 sufficient conditions as functions of $\rho$. 


\subsection{No arbitrage in the Long Term Heston SVI approximation}

In this section, we look at the Long Term Heston SVI introduced by Gatheral and Jacquier in 2 . The authors prove that, under an appropriate hypothesis on the Heston parameters, the Heston implied volatility converges to an SVI implied volatility when the time-to-maturity goes to infinity.

In the Heston model, the underlying process $S_{t}$ follows the stochastic differential equation of the form

$$
\begin{aligned}
d S_{t} & =\sqrt{v_{t}} S_{t} d W_{t}, & & S_{0}>0 \\
d v_{t} & =\tilde{\kappa}\left(\tilde{\theta}-v_{t}\right) d t+\tilde{\sigma} \sqrt{v_{t}} d Z_{t}, & & v_{0}>0 \\
<W, Z>_{t} & =\tilde{\rho} d t . & &
\end{aligned}
$$

We use the $\sim$ hat to distinguish Heston parameters from SVI and SSVI parameters.

Recall the canonical SSVI equation eq. 222. The authors show that setting

$$
\begin{aligned}
\theta & :=\frac{4 \tilde{\kappa} \tilde{\theta} T}{\tilde{\sigma}^{2}\left(1-\tilde{\rho}^{2}\right)}\left(\sqrt{(2 \tilde{\kappa}-\tilde{\rho} \tilde{\sigma})^{2}+\tilde{\sigma}^{2}\left(1-\tilde{\rho}^{2}\right)}-(2 \tilde{\kappa}-\tilde{\rho} \tilde{\sigma})\right), \\
\varphi & :=\frac{\tilde{\sigma}}{\tilde{\kappa} \tilde{\theta} T},
\end{aligned}
$$

the Heston model converges to an SVI with parameters

$$
a=\frac{\theta}{2}\left(1-\rho^{2}\right), \quad b=\frac{\theta \varphi}{2}, \quad \rho=\tilde{\rho}, \quad m=-\frac{\rho}{\varphi}, \quad \sigma=\frac{\sqrt{1-\rho^{2}}}{\varphi} .
$$

Observe that it holds $\gamma=\sqrt{1-\rho^{2}}$ and $\mu=-\frac{\rho}{\sqrt{1-\rho^{2}}}=l^{*}$. In this way it is evident that the Long Term Heston corresponds to a sub-SVI with three parameters and, in particular, it corresponds to an SSVI!

We already know from Lemma 6.2 that an SSVI always satisfies the Fukasawa conditions. The only requirement needed for a no arbitrage parametrization is $\sigma>\sigma^{*}(b, \rho)$. Then, a Long Term Heston is free of arbitrage iff it satisfies proposition 6.3. Since $\sigma$ depends linearly to $T$, indeed $\sigma=\frac{\tilde{k} \tilde{\theta} \sqrt{1-\rho^{2}}}{\tilde{\sigma}} T$, it increases with $T$. On the other hand, $b$ and $\rho$ are constant with respect to $T$ so $\sigma^{*}(b, \rho)$ is also constant and $\sigma$ will eventually become greater than the arbitrage bound for $T$ going to $\infty$. Then, choosing $T$ large enough will grant no arbitrage in the SSVI Long Term Heston parametrization.

This was intuitively true, since the SSVI Long Term Heston approximates the Heston smile, which is itself arbitrage-free; now the convergence in the proof of Gatheral and Jacquier is pointwise, so the no arbitrage property at a fixed $T$ was still to be proven.

Let us try now to compute a lower bound for $T$ which grants no arbitrage.

Suppose $\rho \geq 0$, then from Remark 6.4 $f$ reaches its supremum on the right of $l_{2}$, the second zero of $g_{2}$. The requirement $\sigma \geq-\frac{b g_{2}(l)}{2\left(h^{2}(l)-b^{2} g^{2}(l)\right)}$ for every $l>l_{2}$ can be rewritten substituting $\sigma$ with its expressions in terms of the Heston parameters, as

$$
T \geq A \sup _{l>l_{2}}-\frac{g_{2}(l)}{h^{2}(l)-b^{2} g^{2}(l)}
$$


with

$$
\begin{aligned}
A & :=\frac{b \tilde{\sigma}}{2 \tilde{\kappa} \tilde{\theta} \sqrt{1-\rho^{2}}}=\left(\sqrt{(2 \tilde{\kappa}-\rho \tilde{\sigma})^{2}+\tilde{\sigma}^{2}\left(1-\rho^{2}\right)}-(2 \tilde{\kappa}-\rho \tilde{\sigma})\right) /\left(\tilde{\kappa} \tilde{\theta}\left(1-\rho^{2}\right)^{\frac{3}{2}}\right), \\
b & =\frac{2}{\tilde{\sigma}\left(1-\rho^{2}\right)}\left(\sqrt{(2 \tilde{\kappa}-\rho \tilde{\sigma})^{2}+\tilde{\sigma}^{2}\left(1-\rho^{2}\right)}-(2 \tilde{\kappa}-\rho \tilde{\sigma})\right) .
\end{aligned}
$$

Note than the function of which we look for the supremum does only depend on $\rho$ and on $b$.

The numerator attains its minimum at the locus of the unique minimum of $g_{2}$ on the right of $l_{2}$, that we denoted $m_{2}(\rho)$. We have proven that for SSVI, $h$ is a decreasing function so it is always greater than $h(\infty)=\frac{1}{2}$, while $g$ is increasing with limit $g(\infty)=\frac{(1+\rho)}{4}$. In this way, for $T \geq-\frac{8 b \tilde{\sigma} g_{2}\left(m_{2}(\rho)\right)}{\tilde{\kappa} \tilde{\theta} \sqrt{1-\rho^{2}}\left(4-b^{2}(1+\rho)^{2}\right)}$, the inequality eq. 33 is satisfied.

In the case $\rho<0$, from Proposition 2.6 it follows that the previous discussion still holds substituting $\rho$ with $-\rho$.

We summarize our findings in the following:

Proposition 6.8 (No arbitrage sub-domain for the Long Term Heston SVI). The Long Term Heston SVI approximation defined by eq. (32) is an SSVI. There is no Butterfly arbitrage in the Long Term Heston SVI approximation as soon as

$$
T \geq-\frac{8 b \tilde{\sigma} g_{2}\left(m_{2}(|\rho|),|\rho|\right)}{\tilde{\kappa} \tilde{\theta} \sqrt{1-\rho^{2}}\left(4-b^{2}(1+|\rho|)^{2}\right)}
$$

where $m_{2}(\rho)$ is the only positive point of minimum of $g_{2}(\cdot, \rho)$ and

$$
b=\frac{2}{\tilde{\sigma}\left(1-\rho^{2}\right)}\left(\sqrt{(2 \tilde{\kappa}-\rho \tilde{\sigma})^{2}+\tilde{\sigma}^{2}\left(1-\rho^{2}\right)}-(2 \tilde{\kappa}-\rho \tilde{\sigma})\right) .
$$

Observe that this Proposition corresponds to Lemma 6.6 using the Long Term Heston notations. 


\section{A Numerical proof of the uniqueness of the critical point of $\tilde{f}$ in SSVI}

As discussed in section 6.2.1 to prove the uniqueness of the critical point of $\tilde{f}$ for SSVI, it is enough to show that the function $n=J_{1} \frac{d^{2} j_{2}}{d x^{2}}-\frac{d^{2} J_{1}}{d x^{2}} j_{2}$ is positive for every $x>x_{m_{2}}(\rho=1)=\frac{2+\sqrt{10}}{6}$ setting $b=\frac{2}{1+\rho}$.

The check consists into evaluating the target function at $\rho$ spanning from 0 to 0.999 and $x$ spanning from $\frac{2+\sqrt{10}}{6}$ to 0.999 , choosing for each variable 1000 points between the extrema. The algorithm is the following:

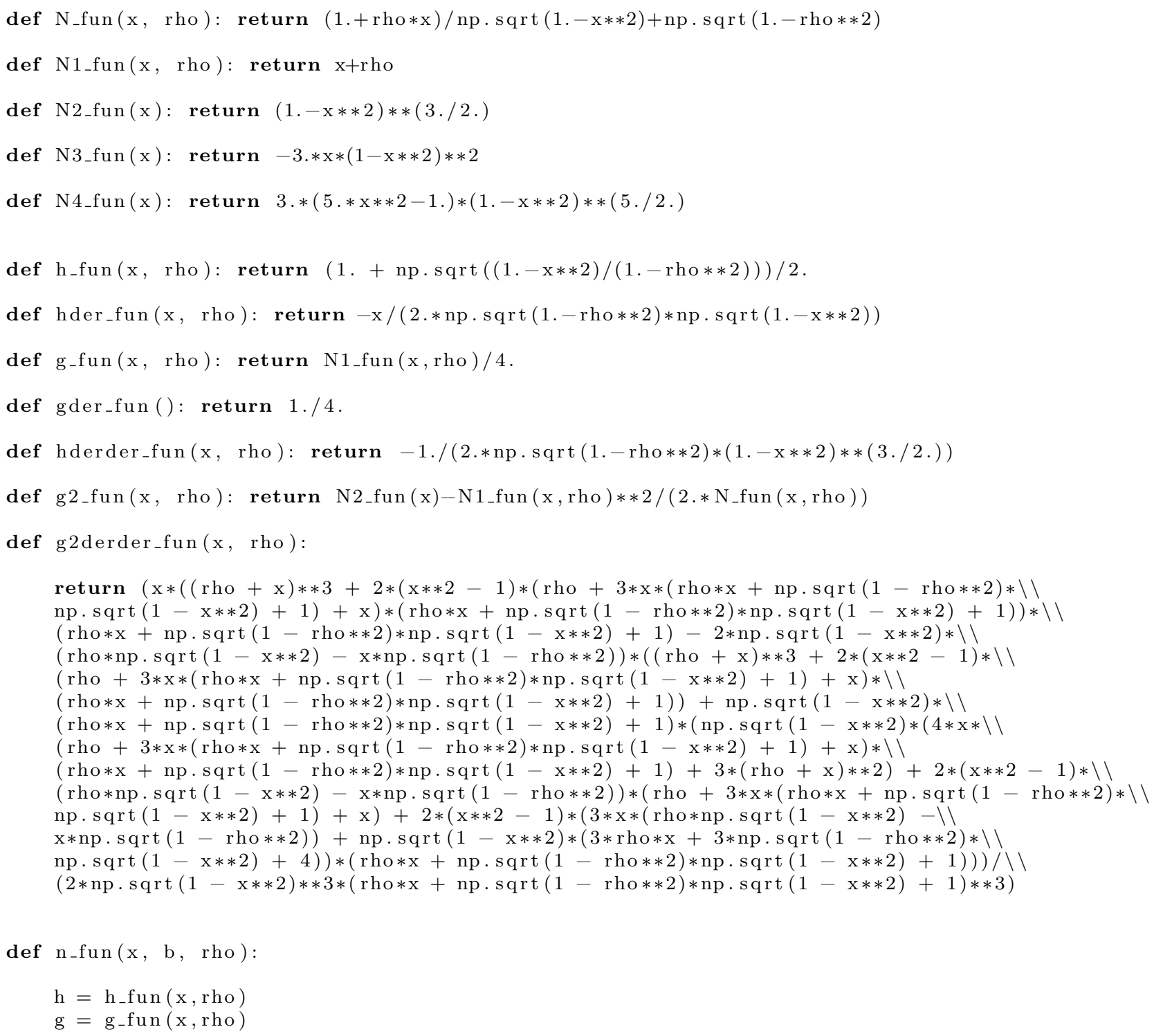




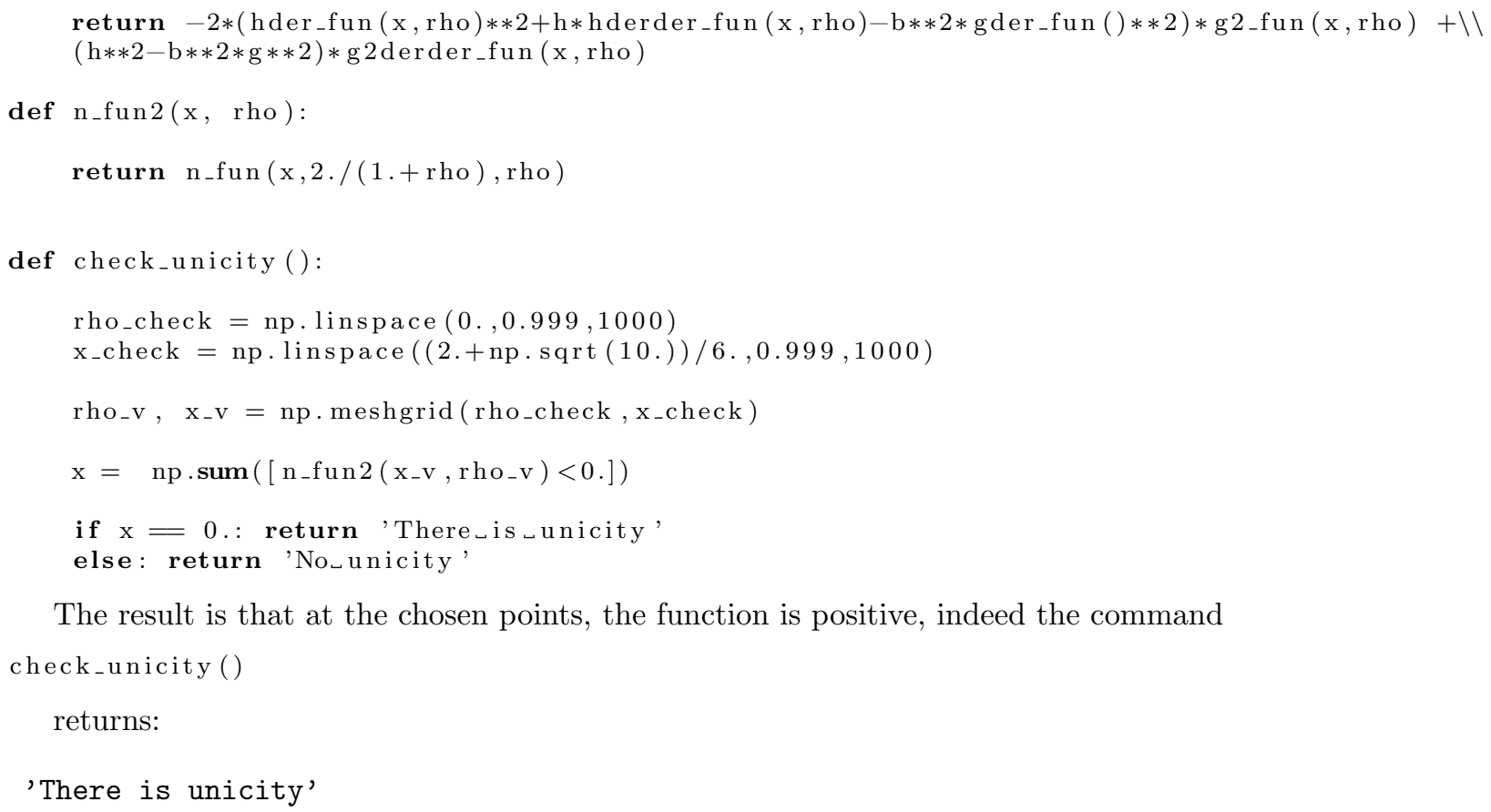

The result is that at the chosen points, the function is positive, indeed the command check_unicity ()

returns:

'There is unicity' 


\section{References}

[1] M. Fukasawa. The normalizing transformation of the implied volatility smile. Mathematical Finance, 22(4):753-762, 2012.

[2] J. Gatheral and A. Jacquier. Convergence of Heston to SVI. Quantitative Finance, 11(8):11291132, 2011.

[3] J. Gatheral and A. Jacquier. Arbitrage-free SVI volatility surfaces. Quantitative Finance, 14(1):59-71, 2014.

[4] G. Guo, A. Jacquier, C. Martini, and L. Neufcourt. Generalized arbitrage-free SVI volatility surfaces. SIAM Journal on Financial Mathematics, 7(1):619-641, 2016.

[5] S. Hendriks and C. Martini. The Extended SSVI Volatility Surface. Journal of Computational Finance, 22(5), 2019.

[6] Claude Martini and Arianna Mingone. No arbitrage svi. arXiv preprint arXiv:2005.03340, 2020.

[7] M. R. Tehranchi. A Black-Scholes inequality: applications and generalisations. Finance and Stochastics, 24(1):1-38, 2020. 